\title{
Tetrahedratic mesophases, chiral order, and helical domains induced by quadrupolar and octupolar interactions
}

\author{
Karol Trojanowski, ${ }^{1}$ Grzegorz Pająk,,${ }^{1,2}$ Lech Longa, ${ }^{1, *}$ and Thomas Wydro ${ }^{1,3, \dagger}$ \\ ${ }^{1}$ Marian Smoluchowski Institute of Physics, Department of Statistical Physics and Mark Kac Center for Complex Systems Research, \\ Jagiellonian University, Reymonta 4, Kraków, Poland \\ ${ }^{2}$ Department of Mathematics and Statistics, University of Strathclyde, Livingstone Tower, Richmond Street, \\ Glasgow, Scotland, United Kingdom \\ ${ }^{3}$ Groupe de Physique Statistique, Institut Jean Lamour, UMR 7198, Nancy-Université, CNRS, Boîte Postale 70239, \\ Boulevard des Aiguillettes, F-54506 Vandoeuvre l'es Nancy Cedex, France
}

(Received 21 March 2012; revised manuscript received 22 May 2012; published 12 July 2012)

\begin{abstract}
We present an exhaustive account of phases and phase transitions that can be stabilized in the recently introduced generalized Lebwohl-Lasher model with quadrupolar and octupolar microscopic interactions [L. Longa, G. Pająk, and T. Wydro, Phys. Rev. E 79, 040701(R) (2009)]. A complete mean-field analysis of the model, along with Monte Carlo simulations allows us to identify four distinct classes of the phase diagrams with a number of multicritical points where, in addition to the standard uniaxial and biaxial nematic phases, the other nematic like phases are stabilized. These involve, among the others, tetrahedratic $(T)$, nematic tetrahedratic $\left(N_{T}\right)$, and chiral nematic tetrahedratic $\left(N_{T}^{*}\right)$ phases of global $T_{d}, D_{2 d}$, and $D_{2}$ symmetry, respectively. Molecular order parameters and correlation functions in these phases are determined. We conclude with generalizations of the model that give a simple molecular interpretation of macroscopic regions with opposite optical activity (ambidextrous chirality), observed, e.g., in bent-core systems. An estimate of the helical pitch in the $N_{T}^{*}$ phase is also given.
\end{abstract}

DOI: 10.1103/PhysRevE.86.011704

PACS number(s): 61.30.Cz, 64.70.mf, 05.70.-a

\section{INTRODUCTION}

Fascinating systems of bent-core (banana-shaped) molecules exhibit a variety of structures, unknown to conventional mesogenic materials [1-4]. Not only do they give rise to a whole family of smectic structures, known as B phases [4], but they also seem to stabilize a nematic phase(s) with a complex supramolecular structure [5,6] including a much sought-after biaxial nematic phase [7-16]. Importantly, a classical way of looking at the liquid crystalline chirality must be revised, as well. While we usually regard chirality and chiral structures of liquid crystals as resulting from the presence of optically active (chiral) molecules (see, e.g., Refs. [17-19]), intrinsically achiral bent-core systems can exhibit macroscopic regions with opposite optical activity, pointing to the occurrence of a spontaneous chiral order. Smectic layers, for example, can become chiral in these systems if the long molecular axes are tilted with respect to the layer normal. Then the smectic layers are spontaneously polarized, normal to the tilt plane, and the three nonequivalent vectors-the polar axis, the tilt direction, and the layer normal - define a right-handed coordinate system and hence introduce the layer chirality, as in the $B 2$ bent-core phase [1]. The layer sense of chirality changes when either polarization direction or tilt direction is reversed. Spontaneous formation of macroscopic chiral domains of opposite handedness in various phases, including isotropic and nematic phases, is also typical of these systems (see, e.g., Ref. [3]).

The observation of this spontaneous chirality leaned toward the hypothesis that the bent-core molecules can acquire tem-

\footnotetext{
*1ech.longa@.uj.edu.pl

†Tomasz.Wydro@ijl.nancy-universite.fr
}

perature weighted conformational chirality of their terminal groups $[1,4,20]$ combined with a strong, short-range molecular ordering of steric and electric dipoles [6,7]. An alternative would be a microphase segregation due to different philicity of various molecular segments in combination with molecular packing as demonstrated for totally rigid, achiral bent-core molecules [21].

Lubensky and Radzihovsky [22] argued that irrespective of the actual microscopic scenario the spontaneously formed chiral structures in bent-core systems can be understood on a macroscopic, phenomenological level by assuming a coupling between octupolar (tetrahedratic) and quadrupolar (biaxial) tensor order parameters. For a complete description of orientational order, a vector order parameter, accounting for a steric or electric, long-range dipolar order, should also be included. Mutual couplings between these three order parameters have been shown to generate a large number of new phases, ranging from the nematic liquids and the tetrahedratic liquid to novel biaxial, polar, and chiral phases. Recent indirect observation of a possible tetrahedratic phase and nematic tetrahedratic phases in a bent-core liquid crystal [23] is an additional support for this scenario. Interestingly, tetrahedratic mesophases along with ambidextrous chiral domains and helical superstructures have also been found in ferrocenomesogens [24].

Although there has been relatively few molecular level studies on this fascinating class of materials the existing work seems consistent with the phenomenological analysis [22,25,26]. In particular, Bisi et al. [27] showed that practically any ensemble of rigid $C_{2}$-symmetric molecules generates a series of competing quadrupolar and octupolar terms to the Onsager's excluded volume, which is prerequisite for having biaxial and tetrahedratic ordering in the mean-field theory. Evaluation of point dispersion interactions [28] between two bent-core molecules gives mathematically similar terms [29]. 
In our recent paper [29] (hereafter referred to as LPW) we introduced a generalized Lebwohl-Lasher model to study orientational ordering in bent-core systems at the microscopic level. The model approximates point dispersion forces between the molecules (or molecular associates) by assuming dominant roles for quadrupolar (uniaxial and biaxial) and octupolar (tetrahedratic) contributions. Results of molecular-field calculations and Monte Carlo computer simulations were carried out to show the simplest scenario possible, i.e., the formation of absolutely stable tetrahedratic, tetrahedratic nematic, and chiral tetrahedratic nematic liquids of global $T_{d}, D_{2 d}$, and $D_{2}$ symmetry, respectively, in addition to the standard uniaxial and biaxial nematic phases. To simplify analysis we disregarded polar interactions between the molecules.

A purpose of this paper is to offer an in-depth analysis of the LPW model. Generalizations of the model, accounting for the observed ambidextrous chirality and helical order, along with an estimate of the pitch in the $N_{T}^{*}$ phase, are also given. The organization of the paper is as follows. In Sec. II, we present a molecular model that comprises lowest-order quadrupolar and octupolar interactions. In Sec. III we then identify molecular order parameters, characterize all absolutely stable orientationally ordered phases, and collect distinct phase diagrams of this model using the mean-field approximation. To extend the mean-field analysis the properties of the model are also determined from Monte Carlo simulations in Sec. IV. In Sec. V we generalize the model by including higher-order couplings between quadrupolar and octupolar tensors and the intermolecular direction. We identify a family of terms responsible for a spatial modulation of chiral structures and give a simple estimate for the helical pitch. We conclude with a short summary in Sec. V.

\section{MODEL}

In our previous paper [29] we introduced a generalized Lebwohl-Lasher dispersion interaction [30-33] that involved the simplest type of coupling between molecular quadrupolar and octupolar degrees of freedom. In the model liquidcrystalline molecules (or molecular complexes) are assumed to interact with the $\mathrm{O}(3)$-invariant pair potential of the form

$$
V(i, j)=-\epsilon\left(r_{i j}\right)\left[\mathbf{Q}\left(\boldsymbol{\Omega}_{i}\right) \cdot \mathbf{Q}\left(\boldsymbol{\Omega}_{j}\right)+\tau \mathbf{T}_{2}^{3}\left(\boldsymbol{\Omega}_{i}\right) \cdot \mathbf{T}_{2}^{3}\left(\boldsymbol{\Omega}_{j}\right)\right],
$$

where $r_{i j}=\left|\mathbf{r}_{i j}\right|$ is the distance between these molecules. The first term represents interactions between two second-rank quadrupolar tensors,

$$
\mathbf{Q}=\mathbf{T}_{0}^{2}+\sqrt{2} \lambda \mathbf{T}_{2}^{2},
$$

defined with respect to twofold axes of the molecular quadrupole moment. The second term accounts for interactions between molecular octupolar, third-rank tensors $\left(\mathbf{T}_{2}^{3}\right)$, defined with respect to twofold axes of the molecular octupole moment. The twofold axes of both tensors are taken to coincide. The $\mathbf{T}_{m}^{L}$ tensors denote symmetry-adapted, real-valued irreducible tensors of angular momentum $L$ in Cartesian form $[34,35]$. Due to the assumed mutual orientation of the twofold axes only three tensors $\mathbf{T}_{m}^{L}$, with $L=2$ and 3 , are relevant:

$$
\begin{aligned}
\mathbf{T}_{0, \mathbf{b}_{k 3}}^{2}\left(\boldsymbol{\Omega}_{k}\right) & \equiv \mathbf{T}_{0}^{2}\left(\boldsymbol{\Omega}_{k}\right)=\sqrt{\frac{3}{2}}\left(\mathbf{b}_{k 3} \otimes \mathbf{b}_{k 3}-\frac{1}{3} \mathbf{1}\right), \\
\mathbf{T}_{2, \mathbf{b}_{k 3}}^{2}\left(\boldsymbol{\Omega}_{k}\right) & \equiv \mathbf{T}_{2}^{2}\left(\boldsymbol{\Omega}_{k}\right)=\frac{1}{\sqrt{2}}\left(\mathbf{b}_{k 1} \otimes \mathbf{b}_{k 1}-\mathbf{b}_{k 2} \otimes \mathbf{b}_{k 2}\right), \\
\mathbf{T}_{2}^{3}\left(\boldsymbol{\Omega}_{k}\right) & =\frac{1}{\sqrt{6}} \sum_{(\mathbf{x}, \mathbf{y}, \mathbf{z}) \in \pi\left(\mathbf{b}_{k 1}, \mathbf{b}_{k 2}, \mathbf{b}_{k 3}\right)} \mathbf{x} \otimes \mathbf{y} \otimes \mathbf{z},
\end{aligned}
$$

where $\boldsymbol{\Omega}_{k}$ is the orthonormal tripod of vectors $\left\{\mathbf{b}_{k 1}, \mathbf{b}_{k 2}, \mathbf{b}_{k 3}\right\}$ attached to the $k$ th molecule (molecular complex), hence defining its orientational degrees of freedom.

The additional index $\mathbf{z}$ in $\mathbf{T}_{m, \mathbf{z}}^{2}$ denotes which vectors of the orthonormal tripod $\{\mathbf{x}, \mathbf{y}, \mathbf{z}\}$ are used to construct $\mathbf{T}_{m}^{2}$. This extended notation will be useful in the analysis of correlation functions. Summation in $\mathbf{T}_{2}^{3}$ runs over six permutations of $\mathbf{b}_{k 1}$, $\mathbf{b}_{k 2}$, and $\mathbf{b}_{k 3}$. The coefficients are chosen such that the tensors of the same rank fulfill the orthogonality condition with respect to the scalar product ".", defined as a full contraction over Cartesian indices

$$
\mathbf{T}_{m}^{L} \cdot \mathbf{T}_{m^{\prime}}^{L^{\prime}}=\left(\mathbf{T}_{m}^{L}\right)_{\alpha \beta \ldots}\left(\mathbf{T}_{m^{\prime}}^{L^{\prime}}\right)_{\alpha \beta \ldots}=\delta_{m m^{\prime}} \delta_{L L^{\prime}} .
$$

The first two tensors in Eq. (3) are used to account for nematic structures. More specifically, the tensors $-\frac{1}{2}\left(\mathbf{T}_{0}^{2}+\right.$ $\left.\sqrt{3} \mathbf{T}_{2}^{2}\right),-\frac{1}{2}\left(\mathbf{T}_{0}^{2}-\sqrt{3} \mathbf{T}_{2}^{2}\right)$, and $\mathbf{T}_{0}^{2}$ are invariant under rotations about $\mathbf{b}_{k 1}, \mathbf{b}_{k 2}$, and $\mathbf{b}_{k 3}$, respectively. Hence, they are $\mathcal{D}_{\infty h}$ symmetric and responsible for the formation of the uniaxial nematiclike order. An arbitrary linear combination of $\mathbf{T}_{0}^{2}$ and $\mathbf{T}_{2}^{2}$ with the exclusion of the above cases gives a biaxial $\mathcal{D}_{2 h^{-}}$ symmetric tensor.

The last tensor, denoted $\mathbf{T}_{2}^{3}$, is the first one in the hierarchy of tensors invariant with respect to tetrahedral point group symmetry $\mathcal{T}_{d}$. The corresponding interaction term, proportional to $\tau$, can be regarded as a higher-order contribution to interaction between, e.g., two ferrocenomesogens, bent-core molecules, or complexes (Fig. 1 in LPW), described in terms of tetrahedratic (nonlinear) point polarizabilities [28].

Special cases of the model (1), where either a quadrupolar type or an octupolar type of the interaction was retained, have already been studied. For $\tau=\lambda=0$ the model reduces to the well-known Maier-Saupe or Lebwohl-Lasher [30] potential, which accounts for isotropic and uniaxial nematic phases connected by a first-order phase transition. The case with $\lambda \neq\{0, \sqrt{3 / 2}\}$ reduces to the model proposed by Luckhurst et al. [31,32] and extensively studied by Biscarini et al. [33]. The model predicts a phase diagram with a prolate uniaxial $N_{U+}$ phase, an oblate uniaxial $N_{U-}$ phase, a biaxial $N_{B}$ phase, and an isotropic $I$ phase, where uniaxial nematic and biaxial nematic phases are connected by a second-order phase transition. A self-dual point, where $\lambda=1 / \sqrt{6}[11,33]$ and where molecules are neither prolate nor oblate, separates a phase in which the biaxial molecules are of the distorted prolate form $(\lambda<1 / \sqrt{6})$ from a phase in which the molecules are of the distorted oblate form $(\lambda>1 / \sqrt{6})$. Further literature on biaxial order for this particular model can be found in Refs. [36,37].

When only the tetrahedratic coupling, proportional to $\epsilon \tau$ is retained in Eq. (1), the model predicts transition from 

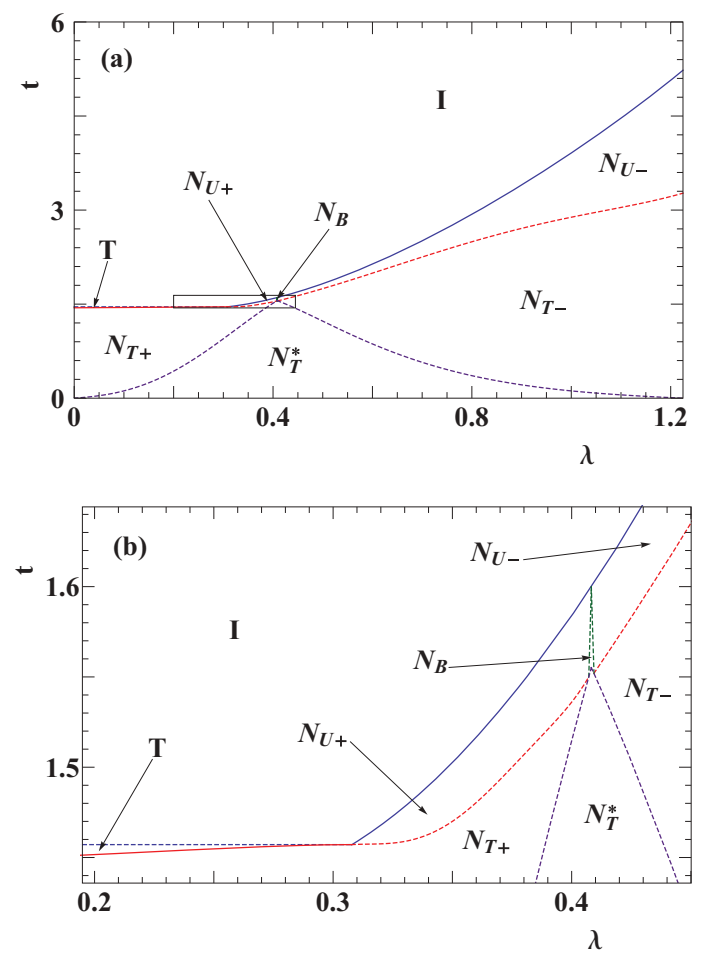

FIG. 1. (Color online) (a) Mean-field phase diagram in $(\lambda, t)$ plane for $\tau=1.7$. A region of stable $N_{B}, T$, and $N_{U+}$ phases, marked with a rectangle, is zoomed in panel (b). Continuous (dashed) lines represent first-order (second-order) phase transitions.

the $I$ phase to the tetrahedratic $(T)$ phase of $\mathcal{T}_{d}$ symmetry, involving an octupolar order parameter. At the microscopic level it was studied by Fel [38,39], by Romano [40], and in LPW. Phenomenological, macroscopic consequences of a tetrahedratic order were discussed in detail by Cladis, Brand, and Pleiner [41-43]. Symmetry classification of orientational structures that combine tensor order parameters of different ranks has been carried out by and Lubensky and Radzihovsky [22] and by Mettout [44].

The combination of biaxial and tetrahedratic interactions, Eq. (1), opens up new possibilities, where the most notable one, as shown in LPW, is the appearance of the chiral nematic tetrahedratic $\left(N_{T}^{*}\right)$ phase of $\mathcal{D}_{2}$ symmetry, in addition to the nematic tetrahedratic phase $\left(N_{T}\right)$ of $\mathcal{D}_{2 d}$ symmetry. A purpose of this paper is to fully explore equilibrium properties of the LPW model. Although we are primarily interested in structures formed by orientational degrees of freedom in the limit when any chirality-induced spatial modulation of the orientational structures can be regarded as a secondary characteristic, we show in Sec. V that in a more complete description the homochiral $N_{T}^{*}$ phase will permit a formation of long-range twist. We also demonstrate that nonchiral structures, including the isotropic liquid, will develop chiral states with ambidextrous chirality.

As in LPW we assume the molecules occupy the sites of a three-dimensional simple cubic lattice. This last assumption, although irrelevant for the mean-field (MF) calculations presented in Sec. III, is kept here mainly for consistency with Monte Carlo simulations, which are carried out to test the MF predictions. The strength of the interaction is given by $\epsilon\left(r_{i j}\right)$, taken to be a positive constant $\epsilon$ when particles $i$ and $j$ are nearest neighbors and zero otherwise. The total interaction potential is thus given by

$$
H=\frac{1}{2} \sum_{\langle i, j\rangle} V(i, j),
$$

with summation running over nearest-neighbor molecular pairs.

The interaction potential (5) with global O(3) symmetry permits spontaneously ordered chiral structures. For a proper thermodynamics description of such structures the $k$ th molecule degrees of freedom, $\boldsymbol{\Omega}_{k}$, should involve a proper rotation $\boldsymbol{\Omega}^{\prime}{ }_{k}$, expressed, e.g., in terms of standard Euler angles $\left(\alpha_{k}, \beta_{k}, \gamma_{k}\right)$ [35] and an inversion operation of the moleculefixed system of frame, referred to as a parity degree of freedom (see LPW). It is quantified in terms of the $p_{k}$ variable, where

$$
p_{k}=\mathbf{b}_{k 1} \cdot\left(\mathbf{b}_{k 2} \times \mathbf{b}_{k 3}\right)= \pm 1 \text {. }
$$

That is,

$$
\boldsymbol{\Omega}_{k}=\left\{p_{k}, \boldsymbol{\Omega}_{k}^{\prime}\right\}
$$

When an inversion is applied to a $k$ th molecular tripod the spherical tensors with odd $L$ 's change sign, in agreement with the general symmetry relation,

$$
\mathbf{T}_{m}^{L}\left(\boldsymbol{\Omega}_{k}\right)=\mathbf{T}_{m}^{L}\left(p_{k}, \boldsymbol{\Omega}_{k}^{\prime}\right)=\left(p_{k}\right)^{L} \mathbf{T}_{m}^{L}\left(\boldsymbol{\Omega}_{k}^{\prime}\right),
$$

where $p_{k}^{2}=1$ and $p_{k}^{3}=p_{k}$. Consequently, the inversions force a sign change of the $\mathbf{T}_{2}^{3}$ tensor in Eq. (1), but leave the $\mathbf{Q}$ tensor unaffected. Finally, since proper rotations and inversions commute, the Ising-like parity degrees of freedom and proper rotations can be treated independently.

As just discussed, associated with each lattice site molecular degrees of freedom involve proper rotations and inversions. Hence, for $\mathrm{O}(3)$-symmetric models the $N$ particle probability density distribution function $P_{N}$ depends on $\left\{p_{i}, \boldsymbol{\Omega}_{i}^{\prime}\right\}, i=1, \ldots, N$. That is, $P_{N}\left(\boldsymbol{\Omega}_{1}, \boldsymbol{\Omega}_{2}, \ldots, \boldsymbol{\Omega}_{N}\right) \equiv$ $P_{N}\left(p_{1}, \boldsymbol{\Omega}^{\prime}{ }_{1}, p_{2}, \boldsymbol{\Omega}^{\prime}{ }_{2}, \ldots, p_{N}, \boldsymbol{\Omega}^{\prime}{ }_{N}\right)$, Eq. (7), with $P_{N} \geqslant 0$ and $\underset{(1, \ldots, N)}{\operatorname{Tr}} P_{N}=1$, where

$$
\underset{(1, \ldots, N)}{\operatorname{Tr}} \equiv \prod_{k=1}^{N}\left(\frac{1}{2} \sum_{p_{k}= \pm 1} \int \mathrm{d} \boldsymbol{\Omega}^{\prime}{ }_{k}\right) .
$$

Now, utilizing the Shannon's formula for entropy the nonequilibrium free energy for the system composed of $N$ molecules interacting through (1) is given by

$$
\beta F_{\text {neq }}=\beta \underset{(1, \ldots, N)}{\operatorname{Tr}}\left[P_{N} H\right]+\underset{(1, \ldots, N)}{\operatorname{Tr}}\left[P_{N} \ln P_{N}\right],
$$

where $\beta=1 /\left(k_{B} T\right)$ is the inverse temperature. Minimizing Eq. (10) with respect to $P_{N}$ gives ordinary statistical mechanics in a canonical ensemble for a system with global $\mathrm{O}(3)$ symmetry. The equilibrium free energy, $F_{\text {eq }}$, is given, as usual, by $F_{\text {eq }}=-\beta^{-1} \ln Z$, where $Z=\underset{(1, \ldots, N)}{\operatorname{Tr}}[\exp (-\beta H)]$. In the next section we explore the equilibrium properties of the model (5) in the mean-field approximation. 


\section{MEAN-FIELD RESULTS}

In mean-field theory we average the interaction energy to obtain an effective single-particle potential due to all the other particles. Mathematically this is equivalent to introducing the one-particle ansatz for $P_{N}$ in Eq. (10):

$$
P_{N}\left(p_{1}, \boldsymbol{\Omega}^{\prime}{ }_{1}, p_{2}, \boldsymbol{\Omega}^{\prime}{ }_{2}, \ldots, p_{N}, \boldsymbol{\Omega}^{\prime}{ }_{N}\right)=\prod_{i=1}^{N} P_{1}\left(p_{i}, \boldsymbol{\Omega}_{i}^{\prime}\right) \equiv \prod_{i=1}^{N} P(i),
$$

which approximates Eq. (10) by the corresponding nonequilibrium MF free energy

$$
\beta F_{\text {neq }}^{\mathrm{MF}}=\frac{1}{2} N d \beta \underset{(1,2)}{\operatorname{Tr}}[P(1) V(1,2) P(2)]+N \underset{(1)}{\operatorname{Tr}}[P(1) \ln P(1)] .
$$

Please note that in MF theory of orientationally and translationally homogeneous liquid structures, where we average the interaction energy to obtain an effective single-particle potential due to all remaining particles, the presence of lattice is reduced to a renormalization of the effective potential by the number of nearest neighbors $(d=6)$ for the simple cubic lattice considered here.

As usual, the equilibrium one-particle distribution function, $P_{\text {eq }}$, is obtained variationally by minimizing $F_{\text {non }}^{\mathrm{MF}}$ with respect to $P(i)$, subject to the normalization condition $\underset{(i)}{\operatorname{Tr}}[P(i)]=1$. The necessary condition for $P_{\mathrm{eq}}, \delta F_{\text {non }}^{\mathrm{MF}} /\left.\delta P\right|_{P=P_{\mathrm{eq}}}=\lambda$, where $\lambda$ is the Lagrange multiplier, becomes reduced to a Fredholmtype nonlinear integral equation,

$$
P_{\mathrm{eq}}(1)=Z_{\mathrm{MF}}^{-1} e^{-\beta d V_{\mathrm{eff}}(1)},
$$

where

$$
\begin{aligned}
V_{\text {eff }}(1) & =\operatorname{Tr}_{(2)}\left[V(1,2) P_{\text {eq }}(2)\right], \\
Z_{\mathrm{MF}} & =\operatorname{Tr}_{(1)}\left[e^{-\beta d V_{\text {eff }}(1)}\right],
\end{aligned}
$$

and where the equilibrium MF free energy becomes

$$
\beta F_{\mathrm{eq}}^{\mathrm{MF}}=-\frac{1}{2} N \beta d \underset{(1)}{\operatorname{Tr}}\left[P_{\mathrm{eq}}(1) V_{\mathrm{eff}}(1)\right]-N \ln Z_{\mathrm{MF}} .
$$

With the assumed choice of mutual orientation of twofold axes of the molecule's $\mathbf{Q}$ and $\mathbf{T}$ tensors six stable structures (Fig. 2 in LPW) are identified among stationary solutions of Eq. (13): (a) the $\mathrm{O}(3)$-symmetric, isotropic phase $(I)$; (b) the $\mathcal{D}_{\infty h}$-symmetric uniaxial prolate $\left(N_{U+}\right)$ and oblate $\left(N_{U_{-}}\right)$ nematic phases; (c) the $\mathcal{D}_{2 h}$-symmetric biaxial nematic $\left(N_{B}\right)$ phase; (d) the $\mathcal{T}_{d}$-symmetric tetrahedratic ( $T$ ) phase; (e) the $\mathcal{D}_{2 d}$-symmetric, distorted tetrahedratic nematic prolate $\left(N_{T+}\right)$ and oblate $\left(N_{T-}\right)$ phases; and (f) the $\mathcal{D}_{2}$-symmetric, chiral nematic tetrahedratic $\left(N_{T}^{*}\right)$ phase.

An important step in solving Eq. (13) is the identification of the order parameters. This is achieved by expanding the oneparticle distribution function $P(\boldsymbol{\Omega}) \equiv P\left(p, \boldsymbol{\Omega}^{\prime}\right)$ in the series of symmetry-adapted, real, linear combinations of the Wigner's rotation matrices $\mathcal{D}_{m^{\prime} n^{\prime}}^{L}$ [35] and real combinations of $\mathcal{D}_{m n}^{L}$ 's multiplied by the parity degree of freedom, $p$. We denote the symmetry-adapted functions, orthogonal over $\mathrm{O}(3)$, as $p_{s} \Delta_{m n}^{L}$,

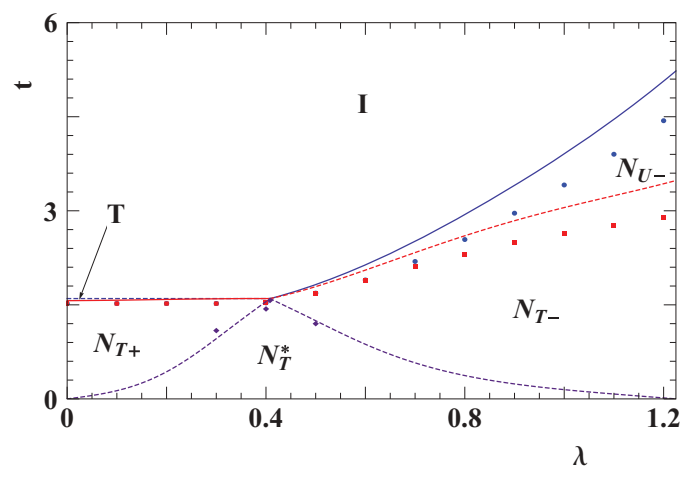

FIG. 2. (Color online) Phase diagram for $\tau=\frac{28}{15}$. Lines represent mean-field results; points are from MC simulations for the threedimensional cubic lattice $(16 \times 16 \times 16)$. Note that all possible phases of the model meet at the Landau point $(\lambda=1 / \sqrt{6}, t=8 / 5)$, which is surprisingly well reproduced in simulations. Continuous (dashed) lines represent first-order (second-order) phase transitions.

where $p_{s} \in\{1, p\}$ and where

$$
\begin{array}{r}
\frac{1}{2} \sum_{p \pm 1} \int d \boldsymbol{\Omega}^{\prime} p_{s} \Delta_{m n}^{(L)}(\boldsymbol{\Omega}) p_{s^{\prime}} \Delta_{m^{\prime} n^{\prime}}^{\left(L^{\prime}\right)}(\boldsymbol{\Omega}) \\
=\frac{8 \pi^{2}}{(2 L+1)} \delta_{L L^{\prime}} \delta_{m m^{\prime}} \delta_{n n^{\prime}} \delta_{s s^{\prime}}
\end{array}
$$

Please observe that, according to Eq. (7), $\Delta$ 's depend on $\boldsymbol{\Omega}$. If the corresponding order parameters are $\overline{p_{s} \Delta_{m n}^{L}}$, where

$$
\overline{\mathbf{X}}=\frac{1}{2} \sum_{p= \pm 1} \int \mathbf{X}\left(p, \boldsymbol{\Omega}^{\prime}\right) P\left(p, \boldsymbol{\Omega}^{\prime}\right) d \mathbf{\Omega}^{\prime} \equiv \operatorname{Tr}[X P],
$$

then the expansion for $P\left(p, \boldsymbol{\Omega}^{\prime}\right)$ can be cast in the following general form:

$$
P\left(p, \boldsymbol{\Omega}^{\prime}\right)=\sum_{L, m, m^{\prime}, s} \frac{2 L+1}{8 \pi^{2}} \overline{p_{s} \Delta_{m m^{\prime}}^{(L)}} p_{s} \Delta_{m m^{\prime}}^{(L)}(\boldsymbol{\Omega}) .
$$

Assuming that the first label $(m)$ in $p_{s} \Delta_{m n}^{L}$ refers to the symmetry of the phase and the second $(n)$ to the molecular symmetry, the following symmetry-adapted functions $p_{s} \Delta_{m n}^{L}$ (or, equivalently, the order parameters $\overline{p_{s} \Delta_{m n}^{L}}$ ) are nonvanishing for each of the previously identified structures.

(a) For the $I$ phase the only nonvanishing function is $\Delta_{00}^{(0)}=1$.

(b) For the $N_{U}$ phases,

$$
\begin{aligned}
\Delta_{m m^{\prime}}^{(L)}= & \left(\frac{1}{\sqrt{2}}\right)^{2+\delta_{0 m}+\delta_{0 m^{\prime}}}\left[\mathcal{D}_{m m^{\prime}}^{L}+(-1)^{L} \mathcal{D}_{m-m^{\prime}}^{L}\right. \\
& \left.+(-1)^{L} \mathcal{D}_{-m m^{\prime}}^{L}+\mathcal{D}_{-m-m^{\prime}}^{L}\right]
\end{aligned}
$$

where $m=0,0 \leqslant m^{\prime} \leqslant L, p_{s}=1$, and where $L$ and $m^{\prime}$ are even. Terms with $m^{\prime}=0$ and $m^{\prime} \neq 0$ correspond to molecular uniaxial and molecular biaxial contributions, respectively. The nonvanishing terms coming from the molecular tetrahedral part 
are

$$
\begin{aligned}
\Delta_{m m^{\prime}}^{(L)}= & \mathcal{N}_{m^{\prime}}^{L}\left(\frac{1}{\sqrt{2}}\right)^{1+\delta_{0 m}}\left[\mathcal{D}_{m m^{\prime}}^{L}+(-1)^{L} \mathcal{D}_{m-m^{\prime}}^{L}\right. \\
& \left.+(-1)^{L} \mathcal{D}_{-m m^{\prime}}^{L}+\mathcal{D}_{-m-m^{\prime}}^{L}\right]+\mathcal{N}_{m^{\prime}}^{L}\left(\frac{1}{\sqrt{2}}\right)^{1+\delta_{0 m}} \\
& \times\left(4 \sum_{m^{\prime \prime}} \mathcal{D}_{m^{\prime \prime} m^{\prime}}^{L}\left(0, \frac{\pi}{2}, 0\right)\left[\mathcal{D}_{-m m^{\prime \prime}}^{L}+(-1)^{L} \mathcal{D}_{m m^{\prime \prime}}^{L}\right]\right),
\end{aligned}
$$

where

$$
\begin{aligned}
\mathcal{N}_{m}^{L}= & \left(2+2 \delta_{0 m}+16(-1)^{L} \mathcal{D}_{m m}^{L}\left(0, \frac{\pi}{2}, 0\right)\right. \\
& \left.+16 \sum_{m^{\prime}} \mathcal{D}_{m m^{\prime}}^{L}\left(0, \frac{\pi}{2}, 0\right) \mathcal{D}_{m^{\prime} m}^{L}\left(0, \frac{\pi}{2}, 0\right)\right)^{-\frac{1}{2}},
\end{aligned}
$$

and where $L$ is even and $m=0$. Tetrahedral conditions must also be fulfilled, which limit $m^{\prime}$ and $m^{\prime \prime}$ to even numbers with $0 \leqslant m^{\prime}, m^{\prime \prime} \leqslant L$. Additionally, the combinations $L+\frac{m^{\prime}}{2}$ and $L+\frac{m^{\prime \prime}}{2}$ must be even for $p_{s}=1$ and odd for $p_{s}=p$.

(c) For the $N_{B}$ phase, the nonvanishing order parameters are given by Eq. (20) with $m$ and $m^{\prime}$ being even and $0 \leqslant m, m^{\prime} \leqslant L$. In addition $L$ must be even for $p_{s}=1$ and odd for $p_{s}=p$. The nonvanishing terms from molecular tetrahedral contribution are of the same form as in (b) except that now the conditions of $L$ being even and $m=0$ are released.

(d) For the $T$ phase the dominant terms come from the molecular $\mathcal{T}_{d}$-symmetric (tetrahedral) part and are given by [27]

$$
\begin{aligned}
\Delta_{m m^{\prime}}^{(L)}= & \mathcal{N}_{m}^{L} \mathcal{N}_{m^{\prime}}^{L}\left(\mathcal{D}_{m m^{\prime}}^{L}+(-1)^{L} \mathcal{D}_{m-m^{\prime}}^{L}+(-1)^{L} \mathcal{D}_{-m m^{\prime}}^{L}+\mathcal{D}_{-m-m^{\prime}}^{L}\right)+\mathcal{N}_{m}^{L} \mathcal{N}_{m^{\prime}}^{L}\left(4 \sum_{m^{\prime \prime}} \mathcal{D}_{m^{\prime \prime} m^{\prime}}^{L}\left(0, \frac{\pi}{2}, 0\right)\left[\mathcal{D}_{-m m^{\prime \prime}}^{L}+(-1)^{L} \mathcal{D}_{m m^{\prime \prime}}^{L}\right]\right) \\
& +\mathcal{N}_{m}^{L} \mathcal{N}_{m^{\prime}}^{L}\left(4 \sum_{m^{\prime \prime}} \mathcal{D}_{m^{\prime \prime} m}^{L}\left(0, \frac{\pi}{2}, 0\right)\left[\mathcal{D}_{-m^{\prime \prime} m^{\prime}}^{L}+(-1)^{L} \mathcal{D}_{-m^{\prime \prime}-m^{\prime}}^{L}\right]\right) \\
& +\mathcal{N}_{m}^{L} \mathcal{N}_{m^{\prime}}^{L}\left(16(-1)^{L} \sum_{m^{\prime \prime}, m^{\prime \prime \prime}} \mathcal{D}_{m^{\prime \prime} m}^{L}\left(0, \frac{\pi}{2}, 0\right) \mathcal{D}_{m^{\prime \prime \prime} m^{\prime}}^{L}\left(0, \frac{\pi}{2}, 0\right) \mathcal{D}_{-m^{\prime \prime} m^{\prime \prime \prime}}^{L}\right)
\end{aligned}
$$

Here $0 \leqslant m, m^{\prime}, m^{\prime \prime}, m^{\prime \prime \prime} \leqslant L$, where $m, m^{\prime}, m^{\prime \prime}$, and $m^{\prime \prime \prime}$ are even. In addition $L+\frac{m}{2}, L+\frac{m^{\prime}}{2}, L+\frac{m^{\prime \prime}}{2}$, and $L+\frac{m^{\prime \prime \prime}}{2}$ are even for $p_{s}=1$ and odd for $p_{s}=p$. Molecular uniaxial and biaxial contributions give rise to the following terms:

$$
\begin{aligned}
\Delta_{m m^{\prime}}^{(L)}= & \mathcal{N}_{m}^{L}\left(\frac{1}{\sqrt{2}}\right)^{1+\delta_{0 m^{\prime}}}\left[\mathcal{D}_{m m^{\prime}}^{L}+(-1)^{L} \mathcal{D}_{m-m^{\prime}}^{L}\right. \\
& \left.+(-1)^{L} \mathcal{D}_{-m m^{\prime}}^{L}+\mathcal{D}_{-m-m^{\prime}}^{L}\right]+\mathcal{N}_{m}^{L}\left(\frac{1}{\sqrt{2}}\right)^{1+\delta_{0 m^{\prime}}} \\
& \times\left(4 \sum _ { m ^ { \prime \prime } } \mathcal { D } _ { - m - m ^ { \prime \prime } } ^ { L } ( 0 , \frac { \pi } { 2 } , 0 ) \left[\mathcal{D}_{-m^{\prime \prime} m^{\prime}}^{L}\right.\right. \\
& \left.\left.+(-1)^{L} \mathcal{D}_{-m^{\prime \prime}-m^{\prime}}^{L}\right]\right)
\end{aligned}
$$

where $m$ and $m^{\prime \prime}$ satisfy the tetrahedral limitations, given right after Eq. (22), while $m^{\prime}$ depends on the symmetry of molecular terms. More specifically, (i) for the purely chiral, $\mathrm{SO}(3)$-symmetric contribution $m^{\prime}=L=0$ and $p_{s}=1$; (ii)

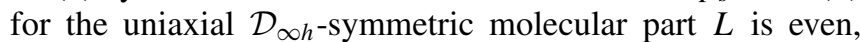
$m^{\prime}=0$, and $p_{s}=1$; (iii) for the biaxial $\mathcal{D}_{2 h}$-symmetric part $m^{\prime}$ is even and $0 \leqslant m^{\prime} \leqslant L$; in addition $L$ is even for $p_{s}=1$ and odd otherwise; (iv) for the $\mathcal{D}_{2 d}$-symmetric molecular part $m^{\prime}$ is even and $0 \leqslant m^{\prime} \leqslant L$; in addition $L+\frac{m^{\prime}}{2}$ is even for $p_{s}=1$ and odd for $p_{s}=p ;(\mathrm{v})$ for the $\mathcal{D}_{2}$-symmetric chiral molecules $m^{\prime}$ is even and $0 \leqslant m^{\prime} \leqslant L$.

(e) For the $N_{T}$ phases the nonvanishing contributions are given by Eq. (20), where $m$ and $m^{\prime}$ are both even and where
$0 \leqslant m, m^{\prime} \leqslant L$. In addition $L+\frac{m}{2}$ is even for $p_{s}=1$ and odd for $p_{s}=p$. The nonvanishing terms from the molecular tetrahedral contribution are the same as those in (b).

(f) For the $N_{T}^{*}$ phase the nonvanishing terms in Eq. (20) are ones with $m$ and $m^{\prime}$ such that $0 \leqslant m, m^{\prime} \leqslant L$. The nonvanishing molecular tetrahedral contributions are the same as those in (c).

The numerical coefficients $\mathcal{D}_{m n}^{L}\left(0, \frac{\pi}{2}, 0\right) \equiv \mathcal{D}_{m n}^{L}\left(\boldsymbol{\Omega}^{\prime}=\right.$ $\left.\left\{0, \frac{\pi}{2}, 0\right\}, p=1\right)$, entering Eqs. (21)-(24), are calculated for right-handed tripods.

Using the expansion (19) one can now express Eqs. (13)(16) in terms of the $\Delta$ functions. The form of the interaction (1) causes that only terms with $L \leqslant 3$ in Eq. (19) are relevant. A simple application of the orthogonality relation (17) along with the expansion (19) allows one to calculate $V_{\text {eff }}$, which can be cast in the following general form

$$
\begin{aligned}
V_{\mathrm{eff}} / \epsilon= & -\left(\overline{\Delta_{00}^{2}}+\sqrt{2} \lambda \overline{\Delta_{02}^{2}}\right)\left(\Delta_{00}^{2}(\boldsymbol{\Omega})+\sqrt{2} \lambda \Delta_{20}^{2}(\boldsymbol{\Omega})\right) \\
& -\left(\overline{\Delta_{20}^{2}}+\sqrt{2} \lambda \overline{\Delta_{22}^{2}}\right)\left(\Delta_{02}^{2}(\boldsymbol{\Omega})+\sqrt{2} \lambda \Delta_{22}^{2}(\boldsymbol{\Omega})\right) \\
& -\tau \overline{\Delta_{22}^{3}} \Delta_{22}^{3}(\boldsymbol{\Omega})
\end{aligned}
$$

Substitution of $V_{\text {eff }}$ back into Eq. (13) reduces that equation to a simpler set of five coupled nonlinear equations. They read

$$
\overline{\Delta_{m n}^{(L)}}=Z_{\mathrm{MF}}^{-1} \underset{(1)}{\operatorname{Tr}}\left[\Delta_{m n}^{(L)}\left(\boldsymbol{\Omega}_{1}\right) e^{-\beta d V_{\text {eff }}(1)}\right],
$$

where the relevant indices $L, m$, and $n$ are those entering Eq. (25). Helpful in deriving the equation above is a general 


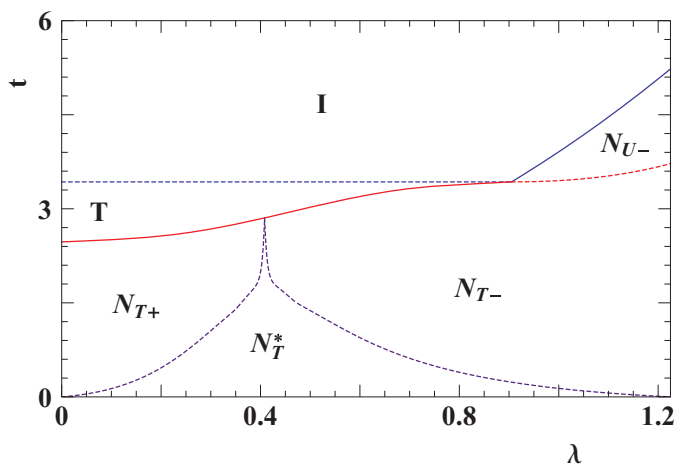

FIG. 3. (Color online) Mean-field phase diagram in the $(\lambda, t)$ plane for $\tau=4$. Note the existence of a critical end-point at $\lambda \approx 0.92$. Continuous (dashed) lines represent first-order (second-order) phase transitions.

relation between $\Delta$ 's and $\mathbf{T}_{m}^{L}$ tensors, given by (see, e.g., Ref. [11])

$$
\Delta_{m m^{\prime}}^{L}\left(\boldsymbol{\Omega}_{i j}\right)=\mathbf{T}_{m}^{L}\left(\boldsymbol{\Omega}_{i}\right) \cdot \mathbf{T}_{m^{\prime}}^{L}\left(\boldsymbol{\Omega}_{j}\right) .
$$

The Eqs. (26) have a rich spectrum of solutions, one of whose corresponds to the isotropic phase $\left.\overline{\left(\Delta_{m n}^{(L)}\right.}=0, L>0\right)$, fulfilling Eq. (26) for all parameters of the model. The solution corresponding to the $N_{U}$ phase, given the director is along the $z$ axis of the laboratory frame, is characterized by nonzero $\overline{\Delta_{0 m}^{2}}$ $(m=0,2)$. In the $N_{B}$ phase only $\overline{\Delta_{22}^{3}}$ vanishes. The solution corresponding to the $N_{T}$ phase is given by nonzero order parameters of the $N_{U}$ phase along with nonzero $\overline{\Delta_{22}^{3}}$. Finally, for the $N_{T}^{*}$ phase all order parameters entering Eq. (25) are nonzero. We found numerically that for the equilibrium state, selected from the above solutions, the MF free energy (16) approaches the absolute minimum.

In our previous paper two limiting classes of the phase diagrams in the $\left[\lambda, t=(\beta \epsilon)^{-1}\right]$ plane were found for fixed values of $\tau$ (LPW, Figs. 3 and 4) using MF calculations and

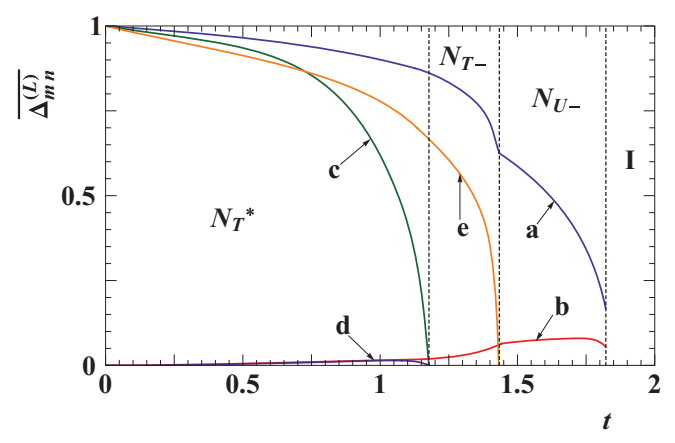

FIG. 4. (Color online) Temperature variation of the order parameters entering parametrization of $V_{\text {eff }}$, Eq. (25), for $\tau=1$ and $\lambda=0.5$ : (a) primary uniaxial order parameter $\overline{\Delta_{00}^{2}}$, (b) secondary uniaxial order parameter $\overline{\Delta_{02}^{2}}$, (c) primary biaxial order parameter $\overline{\Delta_{22}^{2}}$, (d) secondary biaxial order parameter $\overline{\Delta_{20}^{2}}$, and (e) primary tetrahedratic order parameter $\overline{\Delta_{33}^{3}}$.
Monte Carlo (MC) simulations. The first class, represented by the diagram with $\tau=1$, showed no tetrahedratic phase. For the second class of diagrams $(\tau=9)$ the high-temperature region was dominated by the tetrahedratic phase. Some speculations about what can happen for intermediate values of $\tau$ were also given. In particular, recalling the analogy to the dispersion model [33], where $I, N_{U+}, N_{U-}$, and $N_{B}$ phases simultaneously meet at a self-dual (Landau) point, we expected the six phases, $I, T, N_{T+}, N_{T-}, N_{U+}, N_{U-}$, and $N_{B}$, to meet at a self-dual (Landau) point of the present model, which (if it exists) should be found in the phase diagram for $\tau=28 / 15$ and $\lambda=1 / \sqrt{6}[45]$. An alternative scenario would be a first-order $I-N_{T}^{*}$ transition line about $\lambda=1 / \sqrt{6}$. We should add that the critical value $28 / 15$ of $\tau$ has been found by means of the bifurcation analysis of the MF equations (26) about the isotropic phase and confirmed by numerical studies of the MF model.

The present analysis not only supports the existence of a multicritical Landau point but completely characterizes all structures and phase transitions predicted by the model. Generally, most of the phase transitions found are second order except when the uniaxial order parameter $(L=2, m=0$, $p_{s}=1$ ) becomes nonzero. This means that only $I-N_{T}, T-N_{T}$, and $I-N_{U}$ phase transitions are first order. We also find that the previously reported class of the diagrams for $\tau=1$ (LPW, Fig. 3) occurs when $\tau \lesssim 1.54$. The second reported class ( $\tau=9$, LPW, Fig. 4) requires $\tau \gtrsim 6.16$. Intermediate values of $\tau$ give further two classes of the diagrams and one special diagram with a multicritical Landau point.

The first new class of the diagrams is stable for $1.54 \lesssim \tau<$ $28 / 15$. It shows a direct $T-N_{U+}$ first-order phase transition, Fig. 1, in addition to the transitions previously reported in LPW (LPW, Fig. 3). The special diagram is shown in Fig. 2, where six phases coexist at a single multicritical Landau point of $\tau=28 / 15, \lambda=1 / \sqrt{6}$, and $t=8 / 5$. Here occurs a direct second-order phase transition from the isotropic to chiral $N_{T}^{*}$ phase. For $28 / 15<\tau \lesssim 6.16$, Fig. 3 , the biaxial nematic phase is not stable anymore giving rise to the second new class of phase diagrams. Instead of the $N_{B}$ phase the $N_{U-}$ phase is found here along with a bicritical point where two second-order transition lines, $I-T$ and $N_{U-}-N_{T-}$, meet two first-order lines, $I-N_{U-}$ and $T-N_{T-}$. The remaining features of this class of the diagrams have already been accounted for in LPW (LPW, Fig. 4). The critical values 1.54 and 6.16 of $\tau$ are determined numerically from the MF equations, Eqs. (25), (16), and (26), by comparing different transition temperatures. A good starting guess is obtained from a bifurcation analysis of the self-consistent equations (26).

Now we turn to a quantitative analysis of the order parameters. There are three leading (primary) order parameters, $\overline{\Delta_{00}^{2}}$, $\overline{\Delta_{22}^{2}}$, and $\overline{\Delta_{22}^{3}}$, describing various symmetries of stable phases. Along with two secondary order parameters, $\overline{\Delta_{02}^{2}}$ and $\overline{\Delta_{20}^{2}}$, they parametrize $V_{\text {eff }}$, Eq. (25). Their temperature variation across different phases is illustrated in Figs. 4-7.

Besides the order parameters entering $V_{\text {eff }}$ (Figs. 4-7) there are a few secondary order parameters of $L \leqslant 2$, like $\bar{p}$ and $\overline{p \Delta_{m n}^{2}}$, that are of particular interest for the structures identified. These order parameters, with obvious molecular interpretation, become nonzero only when $\overline{\Delta_{22}^{3}} \neq 0$, hence 


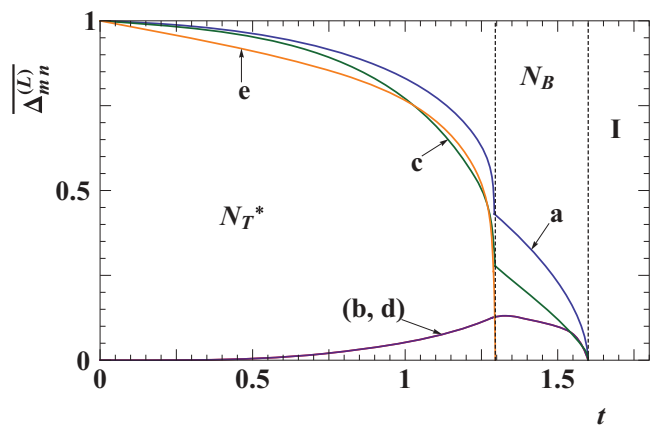

FIG. 5. (Color online) Temperature variation of the order parameters entering parametrization of $V_{\text {eff }}$, Eq. (25), for $\tau=1$ and $\lambda=\frac{1}{\sqrt{6}}$ (for definitions of the labels see the caption to Fig. 4). Note that the secondary order parameters $\overline{\Delta_{02}^{2}}$ and $\overline{\Delta_{20}^{2}}$ coincide.

giving a better insight into the nature of $N_{T}$ and $N_{T}^{*}$ phases. To leading order in $\tau \overline{\Delta_{22}^{3}}$ they read

$\bar{p} \equiv \frac{1}{N} \overline{\sum_{k=1}^{N} p_{k}} \cong \frac{\sqrt{2}}{210 t^{4}} \tau \lambda\left(-3+2 \lambda^{2}\right) \overline{\Delta_{22}^{3}}\left(\overline{\Delta_{20}^{2}}+\sqrt{2} \lambda \overline{\Delta_{22}^{2}}\right)^{3}$,

$\overline{p \Delta_{00}^{2}} \equiv \frac{1}{N} \overline{\sum_{k=1}^{N} p_{k} \Delta_{00}^{2}\left(\boldsymbol{\Omega}_{k}\right)} \cong \frac{\sqrt{2}}{14 t^{2}} \tau \lambda \overline{\Delta_{22}^{3}}\left(\overline{\Delta_{20}^{2}}+\sqrt{2} \lambda \overline{\Delta_{22}^{2}}\right)$,

$\overline{p \Delta_{02}^{2}} \equiv \frac{1}{N} \overline{\sum_{k=1}^{N} p_{k} \Delta_{02}^{2}\left(\boldsymbol{\Omega}_{k}\right)} \cong \frac{-1}{14 t^{2}} \tau \overline{\Delta_{22}^{3}}\left(\overline{\Delta_{20}^{2}}+\sqrt{2} \lambda \overline{\Delta_{22}^{2}}\right)$,

$\overline{p \Delta_{20}^{2}} \equiv \frac{1}{N} \overline{\sum_{k=1}^{N} p_{k} \Delta_{20}^{2}\left(\boldsymbol{\Omega}_{k}\right)} \cong \frac{-\sqrt{2}}{14 t^{2}} \tau \lambda \overline{\Delta_{22}^{3}}\left(\overline{\Delta_{00}^{2}}+\sqrt{2} \lambda \overline{\Delta_{02}^{2}}\right)$,

$$
\overline{p \Delta_{22}^{2}} \equiv \frac{1}{N} \overline{\sum_{k=1}^{N} p_{k} \Delta_{22}^{2}\left(\boldsymbol{\Omega}_{k}\right)} \cong \frac{1}{14 t^{2}} \tau \overline{\Delta_{22}^{3}}\left(\overline{\Delta_{00}^{2}}+\sqrt{2} \lambda \overline{\Delta_{02}^{2}}\right)
$$

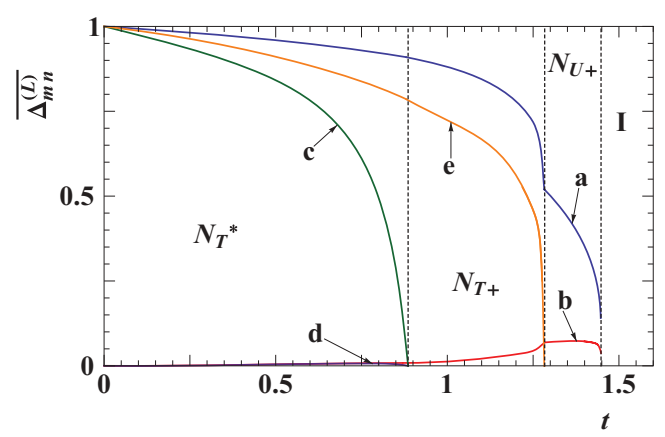

FIG. 6. (Color online) Temperature variation of the order parameters entering parametrization of $V_{\text {eff }}$, Eq. (25), for $\tau=1$ and $\lambda=0.3$ (for definitions of the labels see the caption to Fig. 4). Here prolate uniaxial phases are found.

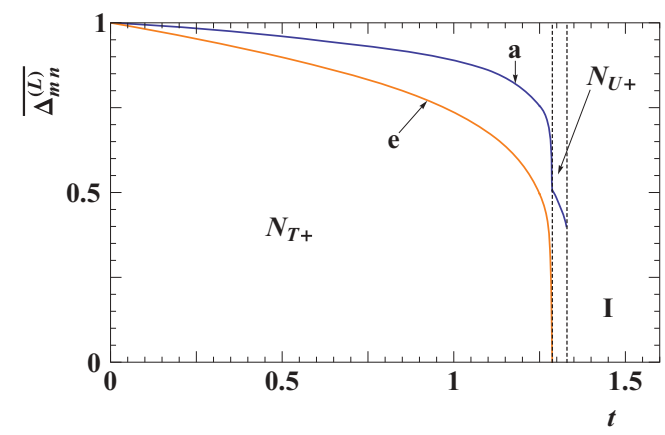

FIG. 7. (Color online) Temperature variation of the order parameters entering parametrization of $V_{\text {eff }}$, Eq. (25), for $\tau=1$ and $\lambda=0$ (for definitions of the labels see the caption to Fig. 4). This corresponds to the case without biaxiality and, hence, only $\overline{\Delta_{00}^{2}}$ (a) and $\overline{\Delta_{22}^{3}}$ (e) are nonzero. The transition temperature to the uniaxial nematic phase has the same value as in the Maier-Saupe model.

The formulas (28)-(32) are derived by identifying $P\left(p, \boldsymbol{\Omega}^{\prime}\right)$ in Eq. (18) with the mean-field distribution function, Eqs. (13) and (25). Next, the resulting formulas are expanded with respect to $\tau \overline{\Delta_{22}^{3}}$ about $\tau \overline{\Delta_{22}^{3}}=0$.

The most apparent conclusion, especially from Eq. (28), is that the $N_{T}^{*}$ phase is stabilized as a result of interplay between octupolar (tetrahedratic) and standard quadrupolar biaxial long-range orders. These, when combined together, lead to a nonvanishing homochiral order as measured by $\bar{p}$, which is absent in the uniaxial limit $\left(\lambda=0\right.$ or $\left.\lambda=\sqrt{\frac{3}{2}}\right)$. Interestingly, in the $N_{T}$ phase, homochiral domains are formed that are weakly biaxial, as exemplified by the secondary order parameters $\overline{p \Delta_{2 m}^{2}}(m=0,2)$. However, these domains are equally populated yielding overall nonchiral structure $(\bar{p}=0)$. Moreover, the secondary biaxial order parameters, Eqs. (31) and (32), are nonzero in the $N_{T}$ phase, where both uniaxial and tetrahedratic orders coexist.

In the next section we compare the MF results with Monte Carlo simulations.

\section{MONTE CARLO SIMULATIONS}

So far, the calculations presented have all been obtained using mean-field theory. Clearly, MF underestimates entropy, which in turn exaggerates the tendency toward ordered phases. In order to assess the validity of the MF theory and account for correlations between molecular degrees of freedom, we perform MC simulations for the model (1). We carry out the simulations on a cubic lattice of relatively large size, $16 \times 16 \times 16$, with periodic boundary conditions, using the standard Metropolis algorithm. The lattice size is chosen such as to limit the fluctuation effects in determining the physical quantities of interest. For the simulations, the orientational degrees of freedom of molecules are coded in a quaternion representation. Each attempted MC move involves proper rotation of a molecule's orientation and parity inversion. The size of the MC rotational step is adjusted to give an acceptance ratio between $30 \%$ and $40 \%$ in the ordered phases. 

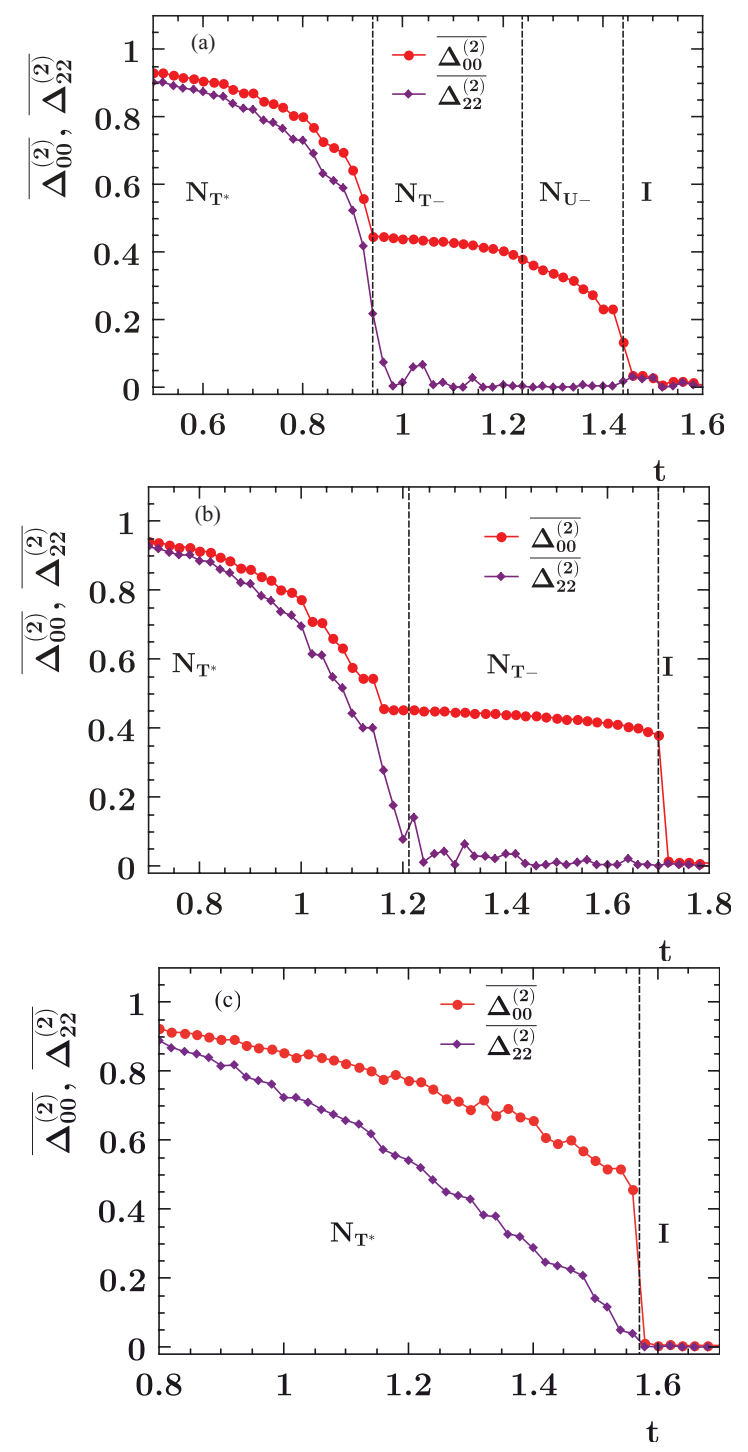

FIG. 8. (Color online) Uniaxial and biaxial order parameters $\overline{\Delta_{00}^{2}}$ and $\overline{\Delta_{22}^{2}}$ for (a) $\tau=1$ and $\lambda=0.5$, (b) $\tau=\frac{28}{15}$ and $\lambda=$ 0.5 , and (c) $\tau=\frac{28}{15}$ and $\lambda=\frac{1}{\sqrt{6}}$. Respective phases and phase transitions are indicated. The order parameters are calculated using the diagonalization method, Eq. (37).

Typically, 50000 to 200000 lattice sweeps are used to thermalize the system and 60000 to 200000 sweeps are used for measurements. Close to a phase transition the temperature is scanned with a step of $\delta t$ down to $\delta t=0.001$. To obtain reliable results in this case, we perform thermalization of up to $10^{6} \mathrm{MC}$ sweeps followed by up to $10^{6} \mathrm{MC}$ cycles of production run. Phase transitions are detected from the peaks of the temperature dependence of specific heat and susceptibilities. In addition, order parameters and pair-correlation functions are determined for uniaxial, biaxial, tetrahedral, and parity degrees of freedom. Detailed simulations are performed for $\tau=1, \frac{28}{15}$ and $\lambda=0.3, \frac{1}{\sqrt{6}}, 0.5$. Some of representative data are shown in Figs. 2 and 8-14.

The calculation of the order parameters is performed by several different approaches. Some are determined from
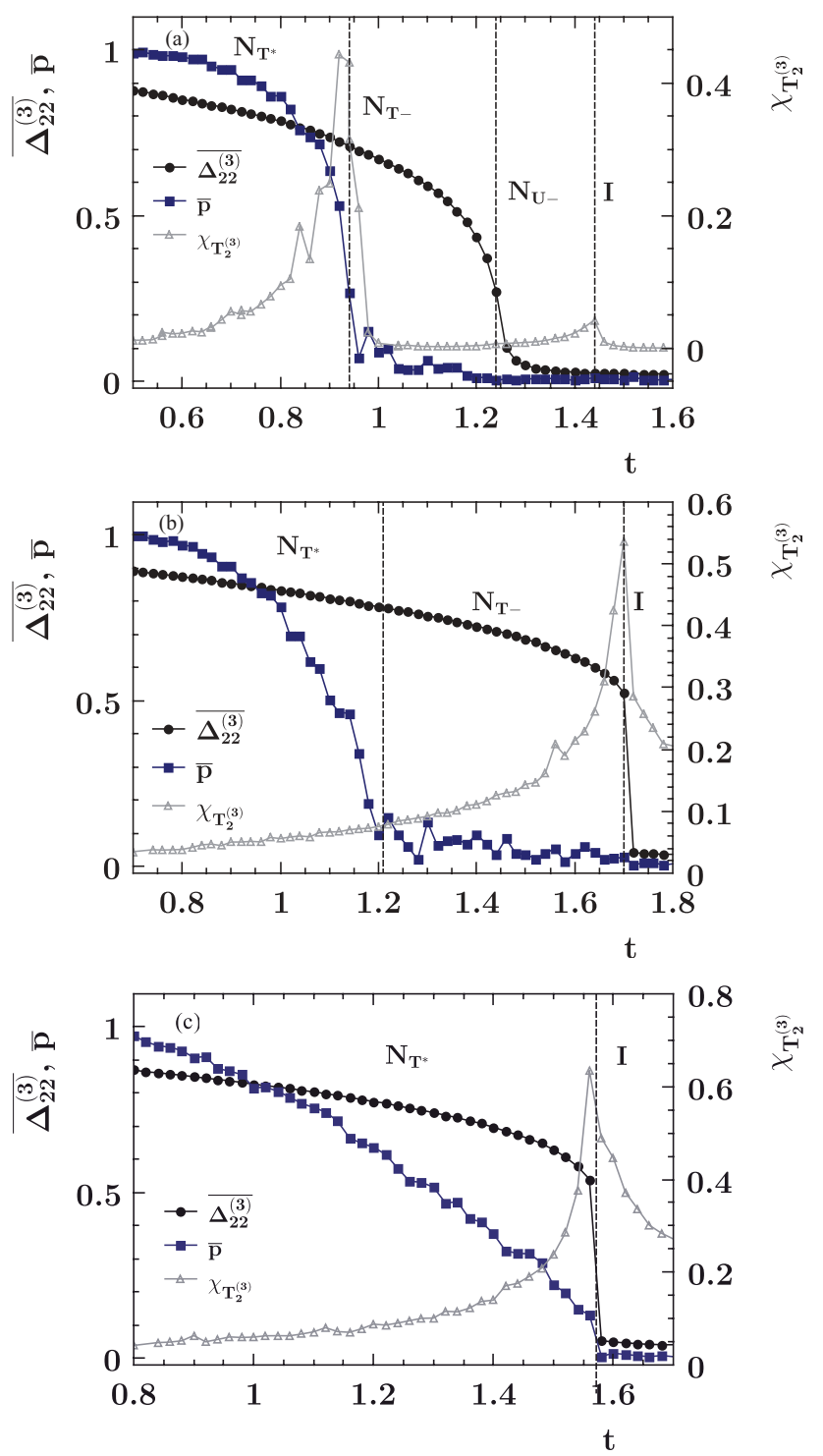

FIG. 9. (Color online) Chirality order parameter $\bar{p}$ and tetrahedratic order parameter $\overline{\Delta_{22}^{3}}$ for (a) $\tau=1$ and $\lambda=0.5$, (b) $\tau=\frac{28}{15}$ and $\lambda=0.5$, and (c) $\tau=\frac{28}{15}$ and $\lambda=\frac{1}{\sqrt{6}}$. Respective phases and phase transitions are indicated.

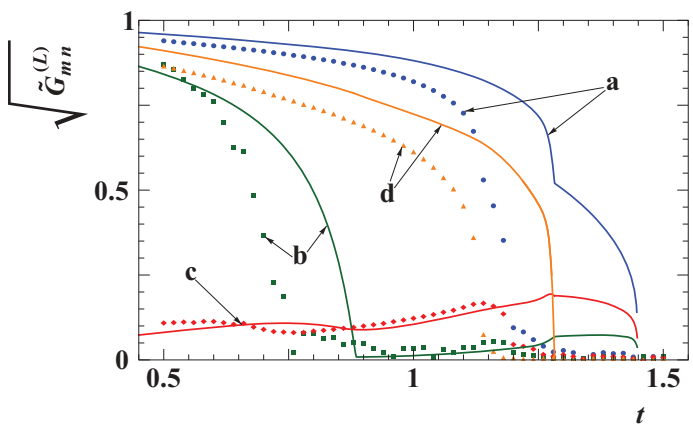

FIG. 10. (Color online) Order parameters from correlation functions for $\tau=1$ and $\lambda=0.3$, where lines represent MF results and points are from MC simulations for the three-dimensional cubic lattice $(16 \times 16 \times 16)$. Curves show square roots of $\tilde{G}_{00}^{2}$ (a), $\tilde{G}_{22}^{2}$ (b), $\tilde{G}_{02}^{2}\left(=\tilde{G}_{20}^{2}\right)(\mathrm{c})$, and $\tilde{G}_{22}^{3}(\mathrm{~d})$. 

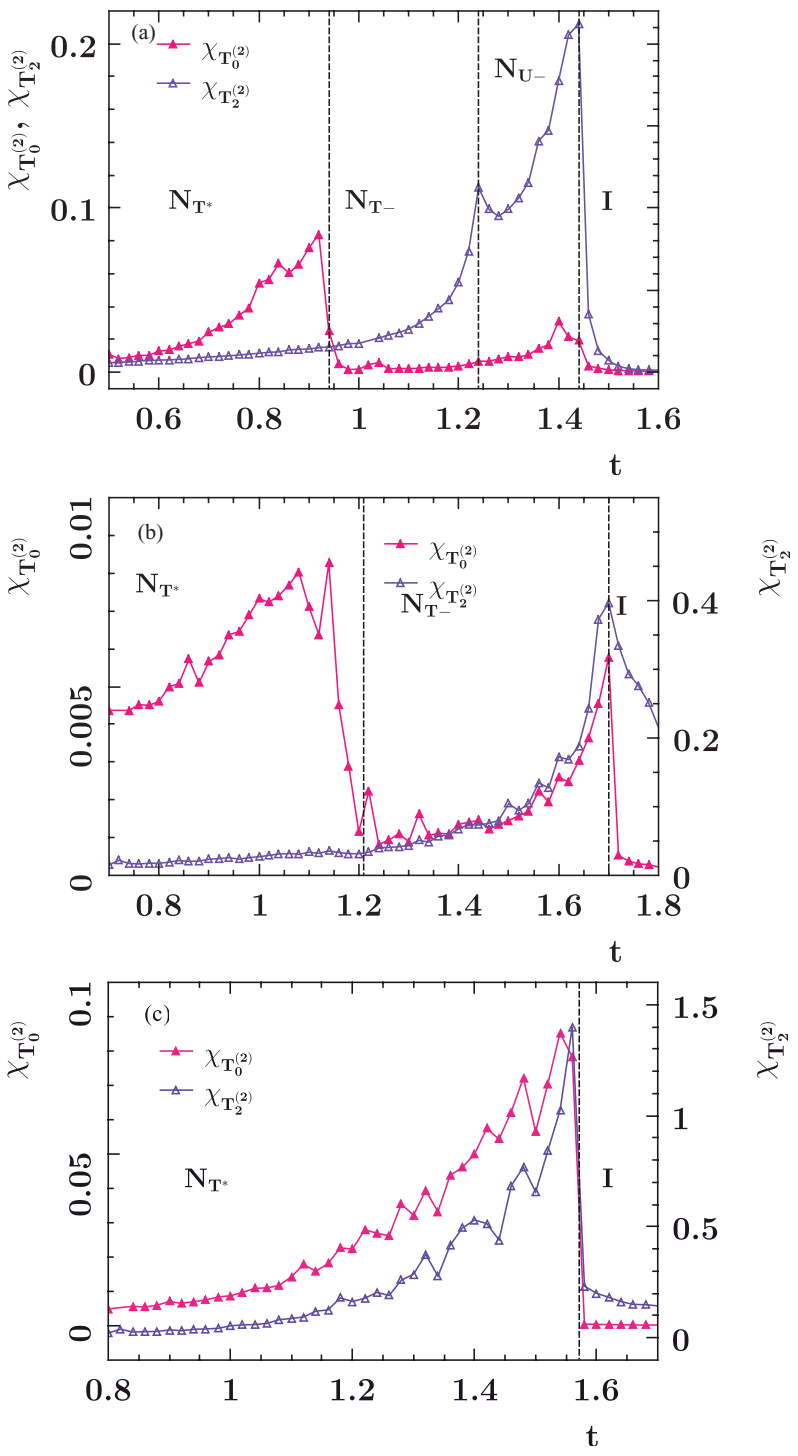

FIG. 11. (Color online) Susceptibilities per molecule for the uniaxial $\left(\chi_{T_{0}^{2}}\right)$ and biaxial $\left(\chi_{T_{2}^{2}}\right)$ tensors: (a) $\tau=1$ and $\lambda=0.5$, (b) $\tau=\frac{28}{15}$ and $\lambda=0.5$, and (c) $\tau=\frac{28}{15}$ and $\lambda=\frac{1}{\sqrt{6}}$. Respective phases and phase transitions are indicated.

the asymptotic behavior of the $\mathrm{O}(3)$-invariant correlation functions

$$
G_{m m^{\prime}, \mathbf{x y}}^{(L)}(|i-j|)=\overline{\mathbf{T}_{m, \mathbf{x}}^{(L)}\left(\boldsymbol{\Omega}_{i}\right) \cdot \mathbf{T}_{m^{\prime}, \mathbf{y}}^{(L)}\left(\boldsymbol{\Omega}_{j}\right)}
$$

and

$$
G_{p p}(|i-j|)=\overline{p_{i} p_{j}} \stackrel{|i-j| \rightarrow \infty}{\longrightarrow} \overline{p_{i} p_{j}}=\bar{p}^{2},
$$

where $\mathbf{x}, \mathbf{y} \in\left(\mathbf{a}_{k}, \mathbf{b}_{k}, \mathbf{c}_{k}\right)$ and $L \leqslant 3$. In order to extract the order parameters from Eq. (33) we note that the unit operator $\mathbf{1}_{\mathcal{G}}$, defined for a given symmetry group $\mathcal{G}$, is given by

$$
\mathbf{1}_{\mathcal{G}}=\sum_{l^{\prime} m^{\prime} s^{\prime}} \mathbf{T}_{m^{\prime}}^{\left(L^{\prime}\right) s^{\prime}} \otimes \mathbf{T}_{m^{\prime}}^{\left(L^{\prime}\right) s^{\prime}},
$$

where $\mathbf{T}_{m^{\prime}}^{\left(L^{\prime}\right) s^{\prime}} \equiv \mathbf{T}_{m^{\prime}, \mathbf{n}}^{\left(L^{\prime}\right) s^{\prime}}$ is constructed using the directors' tripod $\{\mathbf{I}, \mathbf{m}, \mathbf{n}\}$ and where $s^{\prime}$ is the seniority index [34]. Now, with the help of Eq. (35) we can rewrite Eq. (33) using $\Delta_{m n}^{L}$ functions.
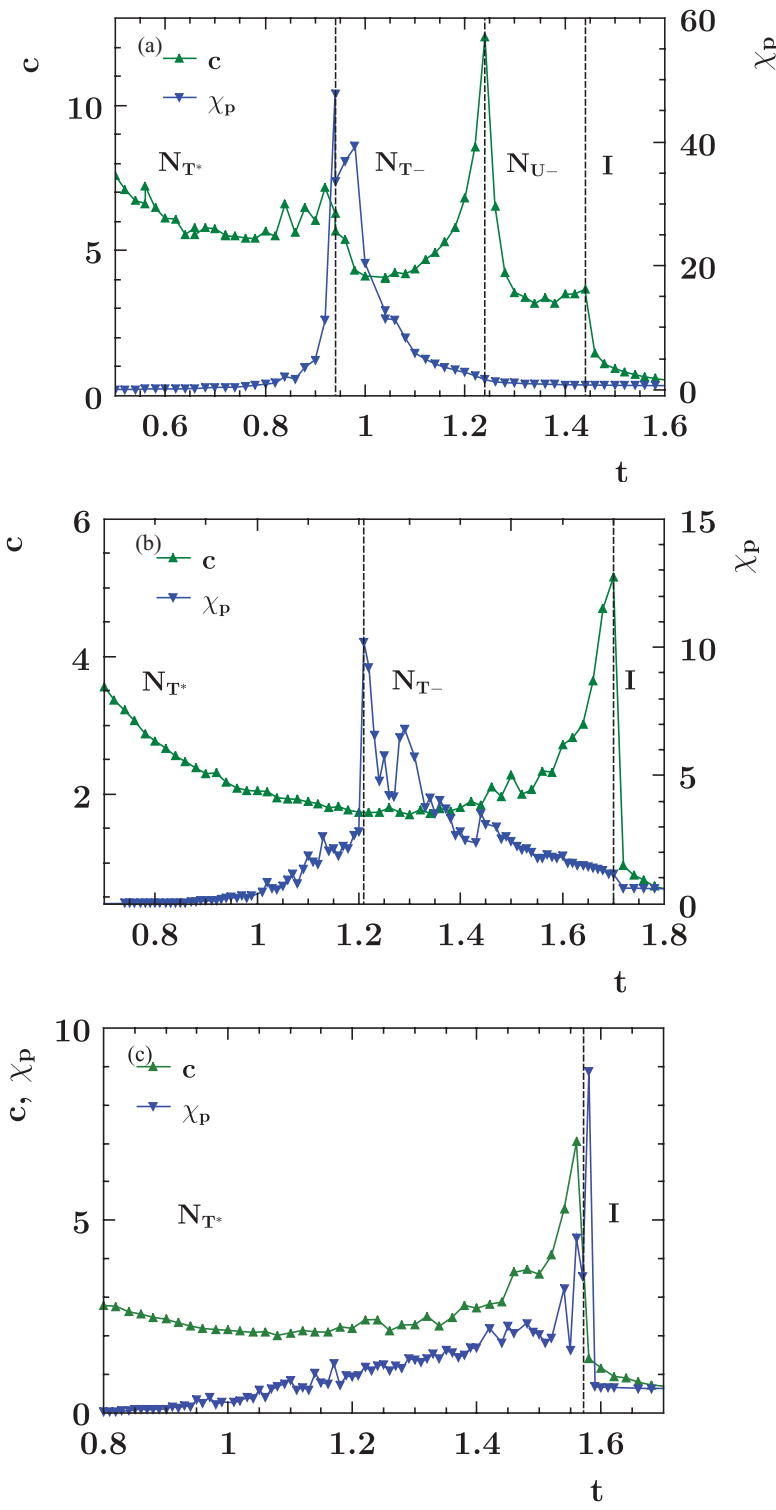

FIG. 12. (Color online) Specific heat $c$ and susceptibility $\chi_{p}$ of the chirality order parameter $\chi_{p}$ for (a) $\tau=1$ and $\lambda=0.5$, (b) $\tau=\frac{28}{15}$ and $\lambda=0.5$, and for (c) $\tau=\frac{28}{15}$ and $\lambda=\frac{1}{\sqrt{6}}$. Respective phases and phase transitions are indicated.

For $L \leqslant 3$, in which case the seniority index is irrelevant, we obtain

$$
\begin{aligned}
G_{m m^{\prime}, \mathbf{x y}}^{(L)}(|i-j|) & =\sum_{m^{\prime \prime}} \overline{\Delta_{m^{\prime \prime} m, \mathbf{x}}^{(L)}\left(\boldsymbol{\Omega}_{i}\right) \Delta_{m^{\prime \prime} m^{\prime}, \mathbf{y}}^{(L)}\left(\boldsymbol{\Omega}_{j}\right)} \\
\stackrel{|i-j| \rightarrow \infty}{\longrightarrow} \tilde{G}_{m m^{\prime}, \mathbf{x y}}^{(L)} & =\sum_{m^{\prime \prime}} \overline{\Delta_{m^{\prime \prime} m, \mathbf{x}}^{(L)}} \overline{\Delta_{m^{\prime \prime} m^{\prime}, \mathbf{y}}^{(L)}}
\end{aligned}
$$

where $m^{\prime \prime}=0,2$ for $L=2$ and $m^{\prime \prime}=2$ for $L=3$. Note that for the tetrahedratic part of $L=3$ we have only one paircorrelation function. This reduction is a direct consequence of the assumed form for the tetrahedratic interaction in Eq. (1). For $L=2$ there are 21 (dependent) correlations of which the asymptotic form $\tilde{G}_{m m^{\prime}, \mathbf{x y}}^{(L)}$ allows one to determine 12 (dependent) order parameters, $\overline{\Delta_{m^{\prime \prime} m, \mathbf{x}}^{(L)}}$. In what follows we limit ourselves to $\mathbf{x}=\mathbf{y}=\mathbf{b}_{k 3}$ and drop the indices $\mathbf{x}$ and $\mathbf{y}$. 

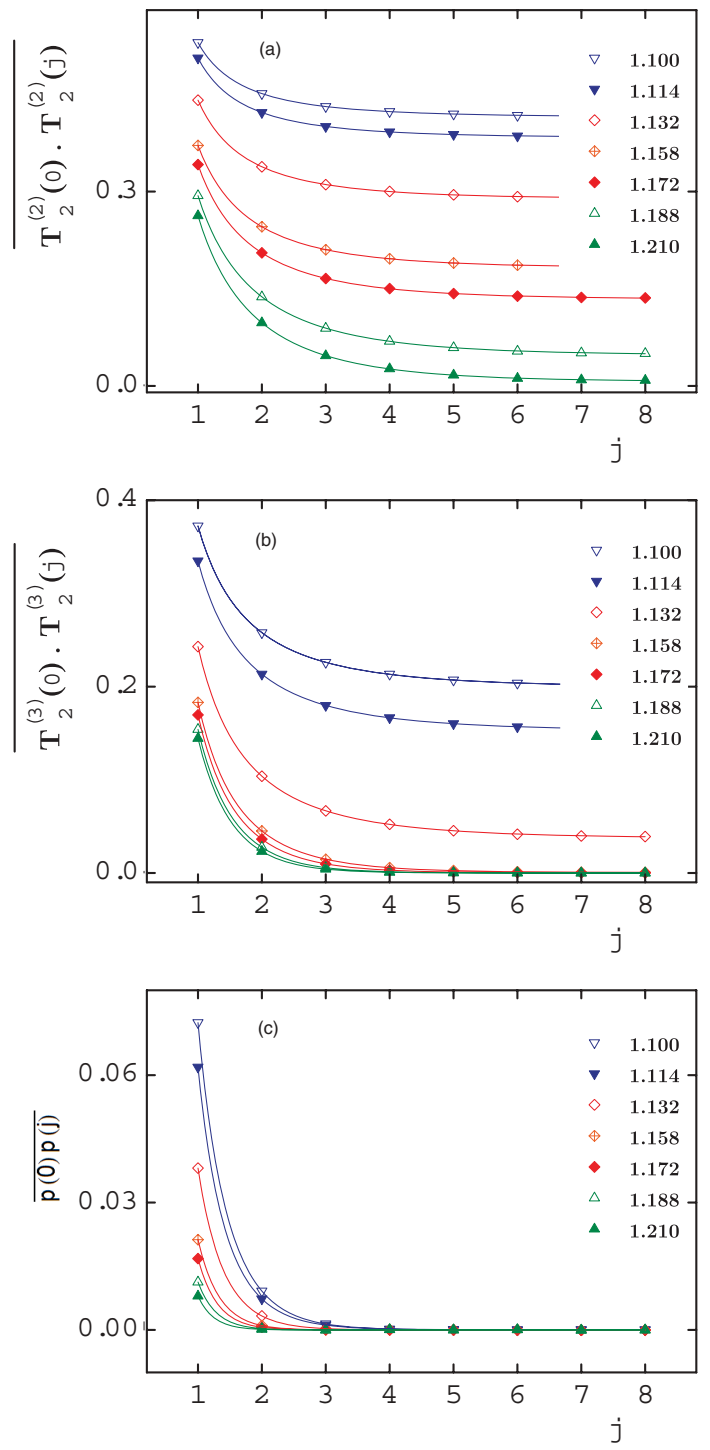

FIG. 13. (Color online) Correlation functions $\overline{\mathbf{T}_{2}^{2}(0) \cdot \mathbf{T}_{2}^{2}(j)}$ (a), $\overline{\mathbf{T}_{2}^{3}(0) \cdot \mathbf{T}_{2}^{3}(j)}$ (b), and $\overline{p(0) p(j)}$ for $\tau=1$ and $\lambda=0.3$. All temperatures (right column) are above the chiral symmetry breaking temperature. The isotropic phase is stable for $t \gtrsim 1.21$; the $N_{U+}$ phase is observed for $1.21 \lesssim t \lesssim 1.114$, and the $N_{T+}$ phase is stable for $1.114 \lesssim t \lesssim 1.1$.

For $L=2$ the expansion in Eq. (36) cannot be resolved for the single-order parameters $\overline{\Delta_{00}^{2}}$ and $\overline{\Delta_{22}^{2}}$. In this case we determine $\bar{\Delta}$ 's by diagonalizing numerically the averaged molecular tensors $\overline{\mathbf{T}_{0}^{2}}$ and $\overline{\mathbf{T}_{2}^{2}}$ to obtain the directors. Then the calculation of $\overline{\Delta_{m^{\prime \prime} m}^{2}}$ follows using the formula

$$
\overline{\Delta_{m^{\prime \prime} m}^{(L)}}=\mathbf{T}_{m^{\prime \prime}, \mathbf{n}}^{(L)} \cdot \overline{\mathbf{T}_{m}^{(L)}} .
$$

Examples of the temperature variation of the order parameters for selected sets of model parameters are given in Figs. 8-10. Shown are $\left\{\overline{\Delta_{00}^{2}}, \overline{\Delta_{22}^{2}}\right\}$ for the $N_{U}$ and $N_{B}$ phases (Fig. 8) and $\left\{\overline{\Delta_{22}^{3}}, \bar{p}\right\}$ for the $N_{T}$ and $N_{T}^{*}$ phases (Fig. 9). A comparison between the MF order parameters and the order parameters determined from the asymptotic behavior of the pair-correlation functions is also given in Fig. 10. Note that
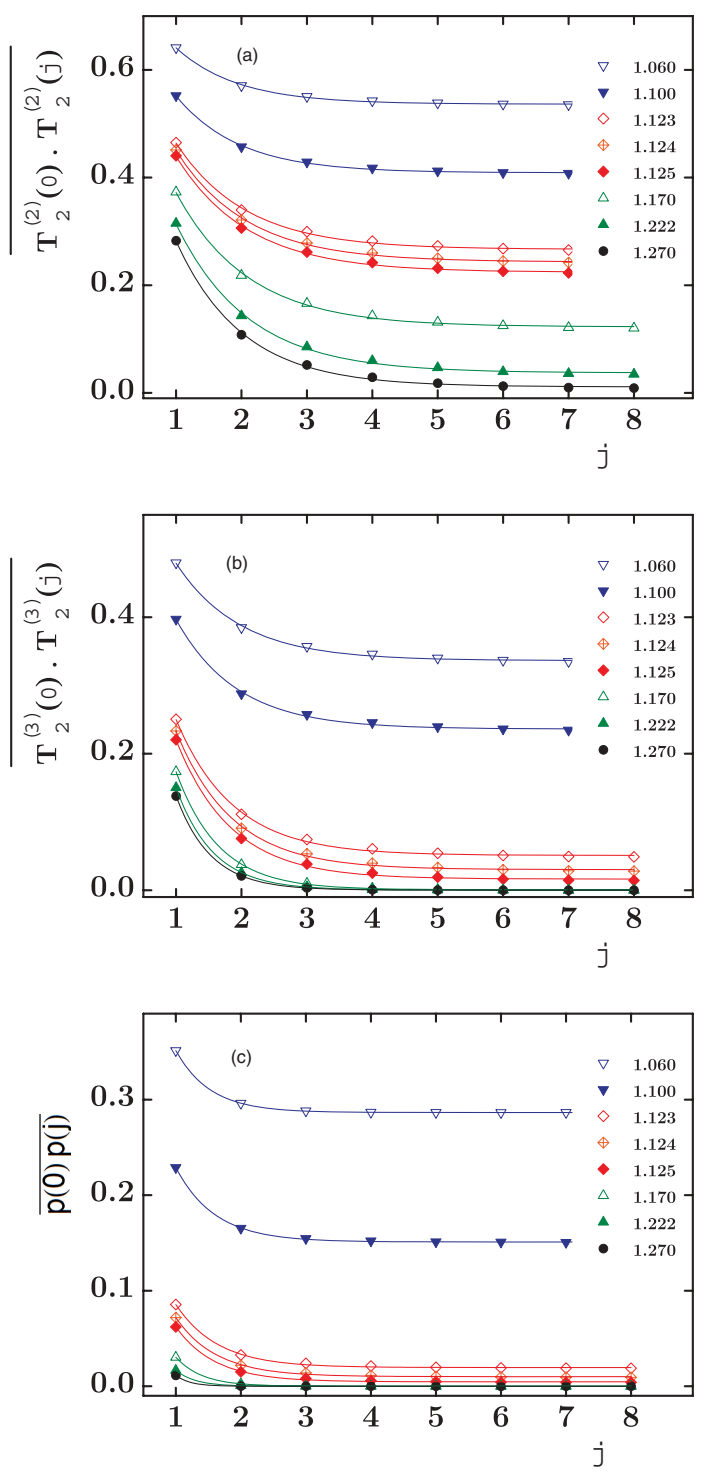

FIG. 14. (Color online) Correlation functions $\overline{\mathbf{T}_{2}^{2}(0) \cdot \mathbf{T}_{2}^{2}(j)}$ (a), $\overline{\mathbf{T}_{2}^{3}(0) \cdot \mathbf{T}_{2}^{3}(j)}$ (b), and $\overline{p(0) p(j)}$ for $\tau=1$ and $\lambda=1 / \sqrt{6}$. The temperatures (right column) correspond to the isotropic phase $(t \gtrsim$ 1.27), the biaxial nematic phase $(1.27 \lesssim t \lesssim 1.123)$, and the $N_{T}^{*}$ phase $(1.123 \lesssim t)$.

for $L=3$ and $m^{\prime \prime}=2$, Eq. (36), we have $\tilde{G}_{22}^{3}=\left(\overline{\Delta_{22}^{3}}\right)^{2}$, so the tetrahedratic order parameter calculated using the correlation method and the diagonalization method should be the same, which is supported by simulations. The order parameter $\bar{p}$ is determined using both the correlation method and the direct average over MC configurations.

The results from simulations are consistent with the MF predictions. In particular, we observe the same sequence of phase transitions with both methods and phase diagrams are similar in shape. As expected, the curves obtained by the MC simulations lie below those obtained from MF. The relative differences between the corresponding transition points depend on the value of the coupling constant $\lambda$ and the type of transition. For the two methods, the relative differences between transition temperatures ranged from about $8 \%$ for $\lambda=0$ and the $N_{U}$-I transition to about $20 \%$ for $\lambda=0.3$ and 
the $N_{T}^{*}-N_{T}$ transition. The most visible difference between the $\mathrm{MF}$ analysis and the MC simulation results occurs at the $N_{U}-\mathrm{I}$ transition below the duality point.

The susceptibility $\chi_{T}$ of a tensorial order parameter $\mathbf{T}(i)$, defined locally on the lattice site $i$, is calculated as a temporal fluctuation of its lattice mean $\mathbf{T}_{L}=\frac{1}{L^{3}} \sum_{i \in \text { Lattice }}^{L^{3}} \mathbf{T}(i)$, where $L$ here denotes the system's size. It reads

$$
\chi_{T}=\overline{T_{L}^{2}}-{\overline{T_{L}}}^{2}
$$

where $T_{L}=\sqrt{\mathbf{T}_{L} \cdot \mathbf{T}_{L}}$. Susceptibilities found in simulations include $\chi_{\mathbf{T}_{0}^{2}}, \chi_{\mathbf{T}_{2}^{2}}, \chi_{\mathbf{T}_{2}^{3}}$, and $\chi_{p}$. Their temperature variations along with that for the specific heat, for $\tau=1, \frac{28}{15}$ and $\lambda=$ $0.5, \frac{1}{\sqrt{6}}$, are found in Figs. 11 and 12 .

For selected $\tau$ and $\lambda$ shown are the temperature variations of $\left\{\chi_{\mathbf{T}_{0}^{2}}, \chi_{\mathbf{T}_{2}^{2}}\right\}$ for the transitions involving uniaxial and biaxial nematic phases (Fig. 11), $\chi_{\mathbf{T}_{2}^{3}}$ (Fig. 9), and $\left\{c, \chi_{p}\right\}$ for the $N_{T}$ and $N_{T}^{*}$ phases (Fig. 12). An example of a full phase diagram determined from simulations is given in Fig. 2.

Generally we observe a good agreement in determining transition temperatures from the behavior of the order parameters, the susceptibilities, and the specific heat, although the uniaxial $\left(\chi_{\mathbf{T}_{0}^{2}}\right)$ and biaxial $\left(\chi_{\mathbf{T}_{2}^{2}}\right)$ susceptibilities [see, e.g., Fig. 11(b)] exhibit, in some cases, considerable fluctuations. Computationally most demanding is a localization of the transition temperature to the $N_{T}^{*}$ phase. This is due to the fact that at low temperatures the thermalization time of the parity degrees of freedom becomes large compared to the thermalization time of purely orientational degrees of freedom. This occurs because the discrete parity switch involves comparatively large changes in local energies as contrasted with purely rotational moves, thereby lowering the number of accepted Metropolis moves. The effect is seen in the behavior of the susceptibility $\chi_{p}$ (Fig. 12).

Computer simulations allow for studies of correlations between molecular degrees of freedom. Three types of correlations, $\overline{\mathbf{T}_{2}^{2}\left(\boldsymbol{\Omega}_{i}\right) \cdot \mathbf{T}_{2}^{2}\left(\boldsymbol{\Omega}_{j}\right)} \equiv \overline{\mathbf{T}_{2}^{2}(0) \cdot \mathbf{T}_{2}^{2}(j-i)}$, $\overline{\mathbf{T}_{2}^{3}\left(\boldsymbol{\Omega}_{i}\right) \cdot \mathbf{T}_{2}^{3}\left(\boldsymbol{\Omega}_{j}\right)} \equiv \overline{\mathbf{T}_{2}^{3}(0) \cdot \mathbf{T}_{2}^{3}(j-i)}, \quad$ and $\quad \overline{p_{i} p_{j}} \equiv$ $\overline{p(0) p(j-i)}$, characteristic of biaxial, tetrahedratic, and chiral orderings are shown in Figs. 13 and 14. Note that local, temperature-dependent chiral correlations are observed across all phases, including the isotropic one. Except for an immediate vicinity of the phase transitions these correlations decay to its asymptotic value for distances of the order of three lattice spacings.

The second-order nature of some of the observed phase transitions impact the decay of correlation functions. In general, we observe a nonexponential decay of the correlations close to the second-order phase transitions, which fit well to mixed, four-parameter, power-exponential function $A x^{-v} \exp (-x / \xi)+B$ with $v \approx 1$, as predicted by Gaussian theory. The nonexponential decay of correlations is especially pronounced for tetrahedral and biaxial correlations, for both $\lambda=0.3$ and $\lambda=\frac{1}{\sqrt{6}}$.

For $\tau=1$ and $\lambda=0.3$, Fig. 13, the considered temperatures extending from $t=1.100$ to $t=1.21$ are above the chiral symmetry-breaking temperature. This is confirmed by the zero value of the asymptotic chiral-chiral correlations, Fig. 13(c).
For $\tau=1$ and $\lambda=\frac{1}{\sqrt{6}}$, Fig. 13, we observe a week $I-N_{B}$ phase transition followed by a strong $N_{B}-N_{T}^{*}$ phase transition.

Finally, neither MF calculations nor MC simulations support the existence of a line of first-order $I-N_{T}^{*}$ phase transitions in the present model. A direct $I-N_{T}^{*}$ phase transition is found only at an isolated Landau point.

\section{TWISTED MOLECULAR CONFIGURATIONS AND AMBIDEXTROUS CHIRALITY}

The class of spatially homogeneous, orientational structures identified so far can be made much richer by supplementing quadrupolar and octupolar interactions, Eq. (1), with further couplings between the molecular tensors $\mathbf{T}_{0}^{2}, \mathbf{T}_{2}^{2}$, and $\mathbf{T}_{2}^{3}$, Eq. (3), or by assuming that the twofold axes of $\mathbf{Q}$ and $\mathbf{T}_{2}^{3}$ do not coincide. We will discuss these interesting cases in our future publication.

In this section we are concerned with yet another important group of interactions, where the $\mathbf{T}$ tensors, Eq. (3), are coupled to the intermolecular unit vector $\hat{\mathbf{r}}_{i j}$. Such couplings, as we show, can superimpose spatially inhomogeneous (shortor long-range) orientational order on the structures already identified. In particular, the $N_{T}^{*}$ phase becomes unstable against spontaneous twist formation. More specifically, assume that $\left(\hat{\mathbf{r}}_{i j}\right)_{\gamma}=-\left(\hat{\mathbf{r}}_{j i}\right)_{\gamma}$ is the $\gamma$ component of the intermolecular unit vector $\mathbf{r}_{i j} / r_{i j}$. The simplest examples of interactions, linear in $\hat{\mathbf{r}}_{i j}$, that can be added to Eq. (1) are

$$
\begin{aligned}
V_{c}\left(p_{i}, \boldsymbol{\Omega}^{\prime}{ }_{i}, p_{j}, \boldsymbol{\Omega}^{\prime}{ }_{j}\right) \\
=\frac{\kappa}{\epsilon}\left[\mathbf{b}_{\alpha i} \cdot\left(\mathbf{b}_{\beta i} \times \mathbf{b}_{\gamma i}\right) Q_{\alpha \nu}\left(\boldsymbol{\Omega}_{i}\right) Q_{\beta \nu}\left(\boldsymbol{\Omega}_{j}\right)\right. \\
\left.\quad-\mathbf{b}_{\alpha j} \cdot\left(\mathbf{b}_{\beta j} \times \mathbf{b}_{\gamma j}\right) Q_{\alpha \nu}\left(\boldsymbol{\Omega}_{j}\right) Q_{\beta \nu}\left(\boldsymbol{\Omega}_{i}\right)\right]\left(\hat{\mathbf{r}}_{i j}\right)_{\gamma}
\end{aligned}
$$

and

$$
\begin{aligned}
& V_{c}^{T}\left(p_{i}, \boldsymbol{\Omega}_{i}^{\prime}, p_{j}, \boldsymbol{\Omega}^{\prime}{ }_{j}\right) \\
& =\frac{\kappa_{T}}{\epsilon}\left[\mathbf{b}_{\alpha i} \cdot\left(\mathbf{b}_{\beta i} \times \mathbf{b}_{\gamma i}\right) T_{2, \alpha \mu \nu}^{3}\left(\boldsymbol{\Omega}_{i}\right) T_{2, \beta \mu \nu}^{3}\left(\boldsymbol{\Omega}_{j}\right)\right. \\
& \left.\quad-\mathbf{b}_{\alpha j} \cdot\left(\mathbf{b}_{\beta j} \times \mathbf{b}_{\gamma j}\right) T_{2, \alpha \mu \nu}^{3}\left(\boldsymbol{\Omega}_{j}\right) T_{2, \beta \mu \nu}^{3}\left(\boldsymbol{\Omega}_{i}\right)\right]\left(\hat{\mathbf{r}}_{i j}\right)_{\gamma} .
\end{aligned}
$$

In the above expressions we have used an Einstein convention for the repeated indices.

The third-rank molecular tensor $\mathbf{b}_{\alpha} \cdot\left(\mathbf{b}_{\beta} \times \mathbf{b}_{\gamma}\right)$, totally antisymmetric in $\{\alpha, \beta, \gamma\}$, that enters Eqs. (39) and (40) is constructed out of the components of the T tensors (3) as

$$
\mathbf{b}_{\alpha} \cdot\left(\mathbf{b}_{\beta} \times \mathbf{b}_{\gamma}\right)=2 \sqrt{2} \sum_{(x, y, z) \in c\{\alpha, \beta, \gamma\}} T_{0, x \mu}^{2} T_{2, y \nu}^{2} T_{2, \mu \nu z}^{3},
$$

where summation runs over cyclic permutations of $\{\alpha, \beta, \gamma\}$ $(c\{\alpha, \beta, \gamma\}=\{(\alpha, \beta, \gamma),(\gamma, \alpha, \beta),(\beta, \gamma, \alpha)\})$.

The interactions (39) and (40), when added separately to $V$, Eq. (1), yield similar qualitative predictions about the system's behavior. We therefore limit ourselves to the discussion of the role played by the $V_{c}$ term, leaving more exhaustive analysis to our forthcoming publication. The new pair interaction potential, $V_{\text {total }}$, is thus given by adding $V$ and $V_{c}$, Eqs. (1) and (39), which gives

$$
\begin{aligned}
& V_{\text {total }}\left(p_{i}, \boldsymbol{\Omega}_{i}^{\prime}, p_{j}, \boldsymbol{\Omega}^{\prime}{ }_{j}, \hat{\mathbf{r}}_{i j}\right) \\
& \quad=V\left(p_{i}, \boldsymbol{\Omega}^{\prime}{ }_{i}, p_{j}, \boldsymbol{\Omega}^{\prime}{ }_{j}\right)+V_{c}\left(p_{i}, \boldsymbol{\Omega}_{i}^{\prime}, p_{j}, \boldsymbol{\Omega}^{\prime}{ }_{j}, \hat{\mathbf{r}}_{i j}\right) .
\end{aligned}
$$


Clearly, $V_{\text {total }}$ is symmetric with respect to the exchange of particles and with respect to $\pi$ rotations about any of twofold axes of the $\mathbf{Q}\left(\mathbf{T}_{2}^{3}\right)$ tensor. The interaction (42) is also invariant with respect to interchange of the molecule-fixed tripods and their parities, and the subsequent replacement of $\left\{p_{i}, p_{j}\right\}$ by $\left\{-p_{i},-p_{j}\right\}$. More specifically

$$
V_{\text {total }}\left(p_{i}, \boldsymbol{\Omega}_{i}^{\prime}, p_{j}, \boldsymbol{\Omega}^{\prime}{ }_{j}, \hat{\mathbf{r}}_{i j}\right)=V_{\text {total }}\left(-p_{j}, \boldsymbol{\Omega}^{\prime}{ }_{j},-p_{i}, \boldsymbol{\Omega}_{i}^{\prime}, \hat{\mathbf{r}}_{i j}\right),
$$

which, along with $V_{c} \sim\left(p_{i}+p_{j}\right)$, allows one to limit $\kappa$ to $\kappa \geqslant 0$. The proportionality of $V_{c}$ to $\left(p_{i}+p_{j}\right)$ and Eq. (43) also imply that $V_{c}$ is relevant given that $p_{i}=p_{j}$. For states of opposite parity $\left(p_{i}=-p_{j}\right)$ the $V_{c}$ term vanishes.

Now we show that for nonvanishing $\kappa$ the homochiral $N_{T}^{*}$ structure becomes unstable against spontaneous twist formation. Twisted configurations of opposite (ambidextrous) chirality will be formed in nonchiral phases. To see this, we find it useful to express $V_{\text {total }}$ in terms of relative Euler angles $\boldsymbol{\Omega}^{\prime}{ }_{i j}=\left\{\alpha_{i j}, \beta_{i j}, \gamma_{i j}\right\}$ that define the rotation carrying the molecule-fixed right-handed frame $\boldsymbol{\Omega}_{i}^{\prime}$ into the molecule-fixed right-handed frame $\boldsymbol{\Omega}^{\prime}{ }_{j}$. We also use spherical coordinates $\left\{\theta_{i j}, \phi_{i j}\right\}$, taken with respect to the $\boldsymbol{\Omega}^{\prime}{ }_{i}$ basis, to express $\hat{\mathbf{r}}_{i j}$ as $\hat{\mathbf{r}}_{i j}=\left\{\sin \left(\theta_{i j}\right) \cos \left(\phi_{i j}\right), \sin \left(\theta_{i j}\right) \sin \left(\phi_{i j}\right), \cos \left(\theta_{i j}\right)\right\}$. This leads to

where

$$
\begin{aligned}
V_{\text {total }}(i, j)= & -\epsilon\left[f_{Q Q}\left(\boldsymbol{\Omega}^{\prime}{ }_{i j}\right)+\tau p_{i} p_{j} f_{T T}\left(\boldsymbol{\Omega}^{\prime}{ }_{i j}\right)\right. \\
& \left.-\kappa\left(p_{i}+p_{j}\right) f_{r}\left(\boldsymbol{\Omega}^{\prime}{ }_{i j}, \theta_{i j}, \phi_{i j}\right)\right],
\end{aligned}
$$

$$
\begin{aligned}
& f_{Q Q}\left(\boldsymbol{\Omega}^{\prime}\right) \\
& =\frac{1}{4}\left\{1+3 \cos (2 \beta)+2 \sqrt{6} \lambda \sin ^{2}(\beta)(\cos (2 \alpha)+\cos (2 \gamma))\right. \\
& \quad+2 \lambda^{2}[\cos (2 \alpha) \cos (2 \gamma)(3+\cos (2 \beta)) \\
& \quad-4 \sin (2 \alpha) \cos (\beta) \sin (2 \gamma)]\} \\
& f_{T T}\left(\boldsymbol{\Omega}^{\prime}\right)=\cos (2 \alpha) \cos (2 \beta) \cos (2 \gamma) \\
& \quad-\frac{1}{8} \sin (2 \alpha) \sin (2 \gamma)[5 \cos (\beta)+3 \cos (3 \beta)] \\
& f_{r}\left(\boldsymbol{\Omega}^{\prime}, \theta, \phi\right) \\
& =\frac{1}{2}\left\{\lambda \sin (2 \alpha) \cos (\theta)\left[\lambda \cos (2 \gamma)(3+\cos (2 \beta))+\sqrt{6} \sin ^{2}(\beta)\right]\right. \\
& +\lambda \sin (2 \gamma)[\sin (\beta) \sin (\theta)(2 \lambda \cos (\alpha+\phi)+\sqrt{6} \cos (\alpha-\phi)) \\
& +4 \lambda \cos (2 \alpha) \cos (\beta) \cos (\theta)]+\frac{1}{2} \sin (2 \beta) \sin (\theta)[\sin (\alpha-\phi) \\
& \quad \times(\sqrt{6} \lambda \cos (2 \gamma)-3)-\lambda \sin (\alpha+\phi)(\sqrt{6}-2 \lambda \cos (2 \gamma))]\}
\end{aligned}
$$

A spatial ordering promoted by $V_{\text {total }}$ can partly be found by studying the ground state properties of Eq. (44). To keep contact with spatially homogeneous but anisotropic phases identified in the preceding sections we carry out energetic considerations for $\epsilon>0$ and $\tau>0$.

If $\kappa=0$, then

$$
\underset{\left\{\Omega^{\prime}\right\}}{\operatorname{Min}}(V(i, j))=-\epsilon\left(1+2 \lambda^{2}+p_{i} p_{j} \tau\right)
$$

is satisfied by a configuration with $p_{i}=p_{j}$ and with twofold axes of $\mathbf{Q}(i)\left(\mathbf{T}_{2}^{3}(i)\right) \mathbf{Q}(j)\left(\mathbf{T}_{2}^{3}(j)\right)$ aligned parallel to each other. This local arrangement can be extended globally over the whole lattice to stabilize the $N_{T}^{*}$ structure.

For $\kappa \neq 0$ and $\lambda$ not too close to its upper uniaxial limit of $\sqrt{3 / 2}$, the ground state configuration is achieved for $\mathbf{b}_{i 1}, \mathbf{b}_{j 1}$,
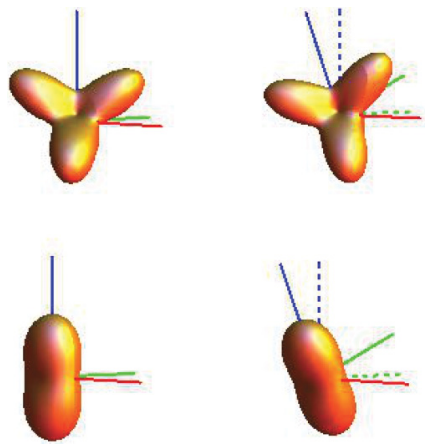

FIG. 15. (Color online) Molecular twist of quadrupoles and octupoles as generated by the interaction potential (42). Parameters taken are $\lambda=0.4$ and $\tau=\kappa=1$.

and $\hat{\mathbf{r}}_{i j}$ being aligned parallel to each other. The perpendicular axes $\left\{\mathbf{b}_{j 2}, \mathbf{b}_{j 3}\right\}$ are rotated by $\beta_{0 i j}$ about $\mathbf{b}_{i 1}$ clockwise $\left(p_{i}+\right.$ $\left.p_{j}=2\right)$ or counterclockwise $\left(p_{i}+p_{j}=-2\right)$ from $\left\{\mathbf{b}_{i 2}, \mathbf{b}_{i 3}\right\}$, where

$$
\begin{gathered}
\tan \left(2 \beta_{0 i j}\right)=\frac{\kappa \Lambda\left(p_{i}+p_{j}\right)}{\Lambda+4 \tau}, \\
\Lambda=3+2 \lambda(\sqrt{6}+\lambda) .
\end{gathered}
$$

An example of mutual orientation of a pair of molecular quadrupoles and octupoles is shown in Fig. 15.

For small $\kappa$ we can expand $\tan \left(2 \beta_{0 i j}\right)$ with respect to $\beta_{0 i j}$ and perform the thermodynamic average of both sides of the obtained approximate relation. This leads to

$$
\overline{\beta_{0}} \equiv \overline{\beta_{0 i j}}=\frac{\kappa \Lambda}{\Lambda+4 \tau} \bar{p},
$$

which is an approximate formula for the averaged molecular twist of the nearest-neighbor tripods in the limit of high orientational order. If we now combine Eq. (51) with the leading mean-field estimate for $\bar{p}$, Eq. (28), we note that at the transition to homochiral $N_{T}^{*}$ structure the molecular configurations will develop a long-range twist of pitch $\overline{\beta_{0}} / \pi$, similar to that observed in cholesteric or blue phases. In the nonchiral phases like $I, N, T$, and $N_{T}$, where $\bar{p}=0$, both populations of left- and right-handed twist configurations will be equally represented leading to what is known, and observed experimentally, as ambidextrous chirality. The effect should be weak [see Eq. (28)] and the studies of the preceding sections correspond to the limit when $\kappa$ [and other similar couplings, like, e.g., Eq. (40)] can be neglected.

\section{CONCLUSIONS}

We have studied equilibrium properties of the recently introduced LPW model with quadrupolar and octupolar pair interactions. The most interesting feature of the model was the appearance of absolutely stable homogeneously chiral nematic tetrahedratic, ordinary nematic tetrahedratic, and tetrahedratic phases. Six qualitatively different classes of the phase diagrams were identified, one of which displayed the existence of a Landau multicritical point where six different phases met. MF results were consistent with MC simulations. 
The inclusion of higher-order pair interactions that involve coupling between the quadrupolar and octupolar tensors and the intermolecular direction induces twist in the $N_{T *}$ phase and yields a simple explanation of recently observed chiral domains of opposite chirality in various phases formed by achiral banana-shaped molecules and ferrocenomesogens. Proposed molecular modeling is generic in the sense that only symmetry allowed terms are retained in the interaction potential. Hence our predictions should hold for any system where tetrahedratic order and quadrupolar order may simultaneously coexist. Orientational structures identified can also coexist with a long-range positional order, characteristic of smectic or crystalline phases.

\section{ACKNOWLEDGMENTS}

This work has been supported by the International $\mathrm{PhD}$ Projects Programme of the Foundation for Polish Science within the European Regional Development Fund of the European Union, Agreement No. MPD/2009/6. One of us (G.P.) acknowledges partial support by the EPSRC (Grant No. EP/H046941/1), which is a part of the Materials World Network Project. The research was carried out with the supercomputer "Deszno" purchased thanks to the financial support of the European Regional Development Fund in the framework of the Polish Innovation Economy Operational Program (Contract No. POIG.02.01.00-12-023/08).
[1] D. R. Link, G. Natale, R. Shao, J. E. Maclennan, N. A. Clark, E. Korblova, and D. M. Walba, Science 278, 1924 (1997).

[2] D. M. Walba, E. Korblova, R. Shao, J. E. Maclennan, D. R. Link, M. A. Glaser, and N. A. Clark, Science 288, 2181 (2000).

[3] R. A. Reddy and C. Tschierske, J. Mater. Chem. 16, 907 (2006).

[4] H. Takezoe and Y. Takanishi, Jpn. J. Appl. Phys. 45, 597 (2006).

[5] O. Francescangeli, F. Vita, C. Ferrero, T. Dingemansc, and E. T. Samulski, Soft Matter 7, 895 (2011).

[6] S. D. Peroukidis, A. G. Vanakaras, and D. J. Photinos, Phys. Rev. E 84, 010702(R) (2011).

[7] J. Peláez and M. R. Wilson, Phys. Rev. Lett. 97, 267801 (2006).

[8] M. S. Park, B. J. Yoon, J. O. Park, V. Prasad, S. Kumar, and M. Srinivasarao, Phys. Rev. Lett. 105, 027801 (2010).

[9] P. Grzybowski and L. Longa, Phys. Rev. Lett. 107, 027802 (2011).

[10] M. A. Osipov and G. Pająk, J. Phys.: Condens. Matter 24, 142201 (2012).

[11] L. Longa, P. Grzybowski, S. Romano, and E. Virga, Phys. Rev. E 71, 051714 (2005).

[12] L. Longa, G. Pająk, and T. Wydro, Phys. Rev. E 76, 011703 (2007).

[13] D. Allender and L. Longa, Phys. Rev. E 78, 011704 (2008).

[14] M. Ciesla and L. Longa, Mol. Cryst. Liq. Cryst. 545, 214 (2011).

[15] Y. Jang, V. P. Panov, A. Kocot, J. K. Vij, A. Lehmann, and C. Tschierske, Appl. Phys. Lett. 95, 183304 (2009).

[16] H. G. Yoon, S. W. Kang, R. Y. Dong, A. Marini, K. A. Suresh, M. Srinivasarao, and S. Kumar, Phys. Rev. E 81, 051706 (2010).

[17] See, e.g., P. G. de Gennes and J. Prost, The Physics of Liquid Crystals, 2nd ed. (Clarendon, Oxford, 1993).

[18] J. Englert, L. Longa, H. Stark, and H.-R. Trebin, Phys. Rev. Lett. 81, 1457 (1998).

[19] L. Longa, M. Cieśla, and H.-R. Trebin, Phys. Rev. E 67, 061705 (2003).

[20] J. Xu, R. L. B. Selinger, J. V. Selinger, and R. Shashidhar, J. Chem. Phys. 115, 4333 (2001).

[21] F. Yan, C. A. Hixson, and D. J. Earl, Phys. Rev. Lett. 101, 157801 (2008).

[22] T. C. Lubensky and L. Radzihovsky, Phys. Rev. E 66, 031704 (2002).
[23] D. Wiant, K. Neupane, S. Sharma, J. T. Gleeson, S. Sprunt, A. Jakli, N. Pradhan, and G. Iannacchione, Phys. Rev. E 77, 061701 (2008).

[24] E. H. Kim, O. N. Kadkin, S. Y. Kim, and M. G. Choi, Eur. J. Inorg. Chem. 2011, 2933 (2011).

[25] H. R. Brand, H. Pleiner, and P. E. Cladis, Phys. A 351, 189 (2005).

[26] H. R. Brand and H. Pleiner, Eur. Phys. J. E: Soft Matter Biol. Phys. 31, 37 (2010).

[27] F. Bisi, L. Longa, G. Pająk, and R. Rosso, Mol. Cryst. Liq. Cryst. 525, 12 (2010).

[28] A. D. Buckingham, Discuss. Faraday Soc. 40, 232 (1965).

[29] L. Longa, G. Pająk, and T. Wydro, Phys. Rev. E 79, 040701(R) (2009).

[30] P. A. Lebwohl and G. Lasher, Phys. Rev. A 6, 426 (1972).

[31] G. R. Luckhurst, C. Zannoni, P. L. Nordio, and U. Segre, Mol. Phys. 30, 1345 (1975).

[32] G. R. Luckhurst and S. Romano, Mol. Phys. 40, 129 (1980).

[33] F. Biscarini, C. Chiccoli, P. Pasini, F. Semeria, and C. Zannoni, Phys. Rev. Lett. 75, 1803 (1995).

[34] J. Jerphagnon, D. Chemla, and R. Bonneville, Adv. Phys. 27, 609 (1978).

[35] D. A. Varshalovich, A. N. Moskalev, and V. K. Khersonskii, Quantum Theory of Angular Momentum (World Scientific, Singapore, 1988).

[36] G. R. Luckhurst, S. Naemura, T. J. Sluckin, K. S. Thomas, and S. S. Turzi, Phys. Rev. E 85, 031705 (2012).

[37] R. Berardi, L. Muccioli, S. Orlandi, M. Ricci, and C. Zannoni, J. Phys.: Condens. Matter 20, 463101 (2008).

[38] L. G. Fel, Phys. Rev. E 52, 702 (1995).

[39] L. G. Fel, Phys. Rev. E 52, 2692 (1995).

[40] S. Romano, Phys. Rev. E 77, 021704 (2008).

[41] P. E. Cladis, H. R. Brand, and H. Pleiner, Liq. Cryst. Today 9, 1 (1999).

[42] H. R. Brand, P. E. Cladis, and H. Pleiner, Europhys. Lett. 57, 368 (2002).

[43] H. R. Brand, P. E. Cladis, and H. Pleiner, Ferroelectrics 315, 165 (2005).

[44] B. Mettout, Phys. Rev. E 74, 041701 (2006).

[45] L. Longa and G. Pająk, Mol. Cryst. Liq. Cryst. 541, 152 (2011). 